\title{
Precipitation variability within an urban monitoring network via microcanonical cascade generators
}

\author{
P. Licznar ${ }^{1}$, C. De Michele ${ }^{2}$, and W. Adamowski ${ }^{3}$ \\ ${ }^{1}$ Faculty of Environmental Engineering, Wroclaw University of Technology, Wrocław, Poland \\ ${ }^{2}$ Department of Civil and Environmental Engineering, Politecnico di Milano, Milan, Italy \\ ${ }^{3}$ Institute of Environmental Engineering, John Paul II Catholic University of Lublin, Stalowa Wola, Poland \\ Correspondence to: P. Licznar (pawel.licznar@pwr.edu.pl)
}

Received: 3 May 2014 - Published in Hydrol. Earth Syst. Sci. Discuss.: 20 May 2014

Revised: 9 December 2014 - Accepted: 11 December 2014 - Published: 26 January 2015

\begin{abstract}
Understanding the variability of precipitation at small scales is fundamental in urban hydrology. Here we consider the case study of Warsaw, Poland, characterized by a precipitation-monitoring network of 25 gauges and microcanonical cascade models as the instrument of investigation.

We address the following issues partially investigated in literature: (1) the calibration of microcanonical cascade model generators in conditions of short time series (i.e., 2.55 years), (2) the identification of the probability distribution of breakdown coefficients (BDCs) through ranking criteria and (3) the variability among the gauges of the monitoring network of the empirical distribution of BDCs.

In particular, (1) we introduce an overlapping moving window algorithm to determine the histogram of BDCs and compare it with the classic non-overlapping moving window algorithm; (2) we compare the 2N-B distribution, a mixed distribution composed of two normal $(\mathrm{N})$ and one beta $(\mathrm{B})$, with the classic B distribution to represent the BDCs using the Akaike information criterion; and (3) we use the cluster analysis to identify patterns of BDC histograms among gauges and timescales.

The scarce representation of the BDCs at large timescales, due to the short period of observation ( $\sim 2.5$ years), is solved through the overlapping moving window algorithm. BDC histograms are described by a $2 \mathrm{~N}-\mathrm{B}$ distribution. A clear evolution of this distribution is observed, in all gauges, from $2 \mathrm{~N}-\mathrm{B}$ for small timescales, $\mathrm{N}-\mathrm{B}$ for intermediate timescales and $\mathrm{B}$ distribution for large timescales.

The performance of the microcanonical cascades is evaluated for the considered gauges. Synthetic time series are analyzed with respect to the intermittency and the variability of
\end{abstract}

intensity and compared to observed series. BDC histograms for each timescale are compared with the 25 gauges in Warsaw and with other gauges located in Poland and Germany.

\section{Introduction}

Urban hydrology requires access to very precise information about the precipitation variability over small spatial and temporal scales. Widespread use of surface runoff models coupled to urban drainage networks increases the common request for rainfall data inputs at high temporal and spatial resolutions. As already estimated a decade ago by Berne et al. (2004), the necessary resolution of rainfall data as the input in hydrological models in Mediterranean regions was about $5 \mathrm{~min}$ in time and $3 \mathrm{~km}$ in space for urban catchments of $\sim 1000$ ha. For smaller urban catchments of $\sim 100$ ha, even higher resolutions of $3 \mathrm{~min}$ and $2 \mathrm{~km}$ were required. Results obtained with the application of operational semidistributed urban hydrology models fully confirmed earlier observations from select study cases in England and France (Gires et al., 2012, 2013). These authors strongly recommend the use of radar data in urban hydrology, especially in the context of real-time control of urban drainage systems. In particular, they opted for $X$ band radars (whose resolution is hectometric), as opposed to the more common $C$ band radars, because they are affected by less uncertainty. Additionally, Gires et al. (2012) stated that small-scale rainfall variability under $1 \mathrm{~km}$ resolution cannot be neglected and should be accounted for in probabilistic ways in the real-time management of urban drainage systems. As a matter of fact, 
the implementation of radar techniques gained a rising popularity in major cities across the EU (for details refer to the Thames Tideway Tunnel (TTT), 2010).

Despite the obvious benefits of radar instruments, radar data are not always available for practical applications. Thus, current versions of even the most advanced computer rainfall-runoff urban drainage models do not consider radar data as rainfall input. Therefore the only possibility of accounting for spatial rainfall variability is to consider different point time series for each subcatchment (Gires et al., 2012). The vast majority of engineering practical calculations and modeling of drainage systems is still associated with point rainfall time series or their elaborations, such as intensityduration-frequency (IDF) curves, depth-duration-frequency (DDF) relations or simplified design hyetographs. This explains the necessity of high temporal resolution of point rainfall measurements in urban catchments. It should be noted that time series at high temporal resolution (1-10 min) with a considerable record length (at least 20-30 years) are nowadays required, especially from the European perspective with respect to the probabilistic assessment of the urban drainage network functioning (Schmitt, 2000; BS EN 752-3, 1997) or the probabilistic assessment of retention volumes at hydraulic-overloaded storm-water systems (Deutsche Vereinigung für Wasserwirtschaft, Abwasser und Abfall e.V., 2006).

The strategy of using local precipitation time series as the basis of the probabilistic assessment of urban drainage systems has two important shortcomings. In the case of local precipitation data shortage, this strategy fails completely. Whereas in all other situations when some local precipitation data sets are accessible, questions and doubts about the representativeness and reliability of data arise. First of all, we consider the doubts regarding the temporal representativeness of data: short data sets could not describe (Willems, 2013) the multi-decadal oscillatory behavior of rainfall extremes in storm-water outflow modeling. Other doubts regarding the spatial representativeness of data include the recording of rainfall time series only in a limited number of gauges installed in selected subcatchments. This results in assigning the same time series to a group of neighboring subcatchments or, in critical but not rare cases, one time series for the whole urban drainage system, habitually collected by a gauge installed near the airport. Sometimes in situations of local precipitation shortage, time series from other locations are allowed by technical guidelines (Schmitt, 2000) only if there is compatibility in terms of annual precipitation totals and IDF values.

Finally, since most of the modeling activity is oriented to predict the future behavior (e.g., in the next 50 years) of drainage systems, the mere use of historical precipitation time series of the last 20-30 years could not be significant to represent the future scenarios. Alternatively, the generation of synthetic time series from precipitation models could represent probable precipitation scenarios to feed hydrodynamic urban drainage models and take into account the uncertainty associated with the discharge. However, it should be pointed out that the information content of historical precipitation records is not increased by precipitation models and synthetic data generation, which just provide an operational basis for the extraction of such information.

Thus, there is a strong motivation for the development of local precipitation models at high temporal resolutions. Many of them are based on the idea of precipitation disaggregation in time. Disaggregation refers to a technique generating consistent rainfall time series at some desired fine timescale (e.g., 5 min resolution) starting from the precipitation at a coarser scale (e.g., daily resolution). At the same time, as stressed by Lombardo et al. (2012), the downscaling techniques aim at producing fine-scale rain time series with statistics consistent with those of observed data. A general overview of rainfall disaggregation methods is given by Koutsoyiannis (2003). Among an ensemble of known techniques, random cascade models, especially microcanonical cascade models (MCMs), are quite often used. The popularity of the latter could be explained by their appeal to engineering applications, the assumption of mass conservation (i.e., rainfall depth conservation) across cascade levels and straight rules for the extraction of cascade generators from local precipitation time series (Cârsteanu and Foufoula-Georgiou, 1996). Olsson (1998), Menabde and Sivapalan (2000), Ahrens (2003) and Paulson and Baxter (2007) provide contributions demonstrating the potentiality of MCMs in rainfall downscaling. Molnar and Burlando (2005) and Hingray and Ben Haha (2005) highlight the application of MCMs in urban hydrology. Hingray and Ben Haha (2005) apply a continuous hydrological simulation to produce from synthetic rainfall series continuous discharge series used afterwards for the retention design. Recently, Licznar (2013) illustrates the possibility of substituting synthetic time series generated from MCMs for observed time series of the probabilistic design of storm-water retention facilities.

Two decades of random cascade applications to precipitation disaggregation has progressed the construction of generators. Quite soon the assumption of independence and identical distribution of the cascade weight generators at all timescales was questioned and found suitable only for a limited, rather narrow range of analyzed scales (Olsson, 1998; Harris et al., 1998). As an alternative, Marshak et al. (1994), Menabde et al. (1997) and Harris et al. (1998) promote the use of the so-called "bounded" random cascade; its weights distribution systematically evolves, decreasing the weights variance with the reduction of timescale. In addition, Rupp et al. (2009) suggest that microcanonical cascade weights should not only be timescale-dependent but also intensitydependent. The common practice of assuming the beta distribution for MCM generators is questioned by Licznar (2011a, b), especially for sub-hourly timescales. Alternatively, MCM generators are assumed normal-beta $(\mathrm{N}-\mathrm{B})$ distributed, with 


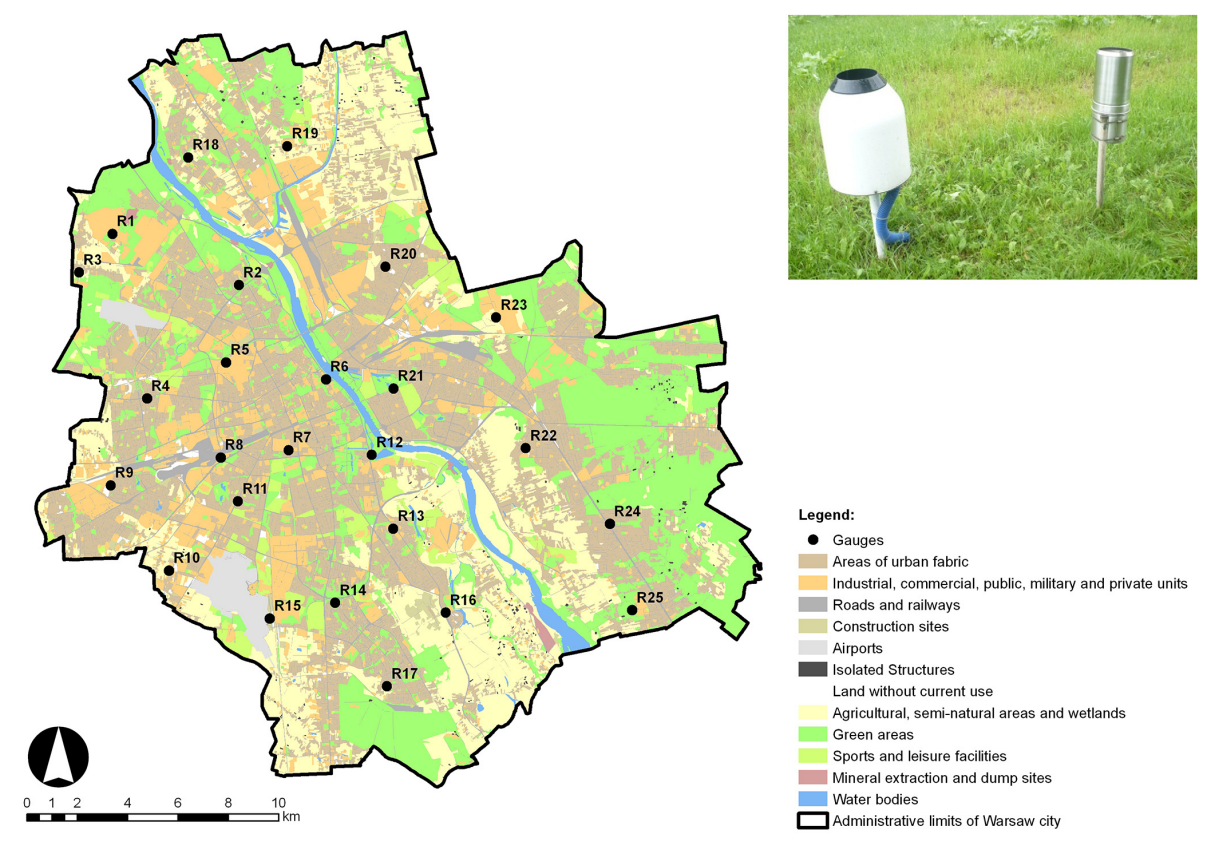

Figure 1. Map of 25 gauges composing the precipitation-monitoring network in Warsaw. Administrative limits of Warsaw city were marked in black. The land use classification was made with the Urban Atlas, which provides pan-European comparable land use and land cover data for large urban zones with more than 100000 inhabitants (http://www.eea.europa.eu/data-and-maps/data/urban-atlas\#tab-metadata). The average density of the network is one instrument for $20.7 \mathrm{~km}^{2}$. MPS weighing-type TRwS 200E gauges were accompanied by standard Hellman gauges for the routine control of daily precipitation totals.

the atom at 0.5 , or $3 \mathrm{~N}-\mathrm{B}$ distributed, composed of three $\mathrm{N}$ and one B distribution. For the sake of clarity, it should be stressed that B refers solely to the distribution of MCM generators and has nothing in common with the $\beta$ model, the simplest cascade model often known as the monofractal model (for details refer to Over and Gupta, 1996).

Molnar and Burlando (2008) explore the variability of MCM generators on a large data set of 10 min time resolution, including 62 stations across Switzerland. These authors investigate seasonal and spatial variability in breakdown distributions to give indications concerning the parameters' estimation of MCM in ungauged locations. To our knowledge, there are only studies considering the large-scale variability (i.e., among different urban areas) of MCM generators, and there is a lack of knowledge concerning the small-scale variability (i.e., within an urban area).

It should be stressed that the fitting of cascade generators was relatively simple but extremely data-demanding. Observational precipitation time series in high resolution usually exceeding 20 years were unavoidable for the fit of the cascade parameters. This resulted in the prevailing practice of comparing the statistics of synthetic and observed time series. In the majority of studies, data originating from old-type manual gauges were subject to obvious uncertainty related to the precision of measurements as well as the resolution of records digitization. Simultaneously, the fitting of theoretical distributions to breakdown coefficients (BDCs) was, in almost all cases, not supported by statistical tests confirming the correctness of achieved results or by the use of some information criteria to rank the theoretical distributions.

Keeping in mind the above discussed needs of urban hydrology, the current state of MCMs and severe limitations of this rainfall disaggregation technique, the goals of our study were the following:

1. Propose a methodology to calibrate microcanonical cascade generators in conditions of short time series;

2. Identify the probability distribution of BDCs through the use of information criterion;

3. Investigate the variability of empirical BDC distributions among a group of gauges;

4. Address the following questions of interest in urban hydrology: is it sufficient to use a single time series for the probabilistic assessment of the entire urban drainage system? Is it sufficient to fit just one MCM for the analysis of the whole city area? Could we continue the practice of supplying urban rainfall-runoff models by time series recorded outside city center by gauges located at the airport or over rural areas? 


\section{Data and methodology}

\subsection{Data}

We used data belonging to a precipitation network of 25 gauges distributed across $517.24 \mathrm{~km}^{2}$ of Warsaw, Poland (Fig. 1). The data set was the same used by Rupp et al. (2012) and consisted of a $1 \mathrm{~min}$ precipitation (both liquid and solid) time series recorded by electronic weighing-type gauges. All stations, TRwS 200E of MPS system Ltd (Fig. 2), were installed and operated by the Municipal Water Supply and Sewerage Company (MWSSC) in Warsaw. Prior to the network installation, studies about the location of the stations were done by the MWSSC to identify the configuration most representative of the precipitation variability within the urban area (Oke, 2006). Finding good places for the installation of gauges was possible due to the fact that the MWSSC in Warsaw operates a vast number of local water intakes and waterand sewage-pumping stations. Due to sanitary standards, all these installations had to occupy terrain with green areas to serve as buffers, e.g., for the spread of odors. In addition, all facilities were fenced and guarded for safety reasons. Therefore all instruments were placed on grass and the neighborhood met at least the requirements of class 2 or 3 as recommended by the WMO (2012). The majority of gauges (i.e., R1, R3, R5, R7, R8, R10, R12, R17, R18 and R19) were able to be installed on flat, horizontal surfaces, surrounded by an open area, thus meeting even requirements for class 1 instruments. In addition, gauge R15 was installed in perfect conditions on the ground at the Warsaw Fryderyk Chopin Airport.

Since the installation of the precipitation network in Warsaw was mainly motivated by the real-time control of the drainage system, all gauges (Fig. 1) were connected to a single data acquisition system. The accuracy of gauge measurements as claimed by the manufacturer was $0.1 \%$, and the data resolution was $0.001 \mathrm{~mm}$ for depth and $1 \mathrm{~min}$ for time. As previously mentioned by Rupp et al. (2012), field tests conducted prior to the operational use of the precipitation network have shown good agreement between simulated and recorded totals and have revealed a dampening/broadening of the input signal evident over the range of a few minutes. The last phenomenon - known as a "step response error" - was studied in detail in laboratory conditions for different gauge types by Lanza et al. (2005). They found that the step error of TRwS gauge was quite small in comparison to other gauges and equal to $3 \mathrm{~min}$ in laboratory conditions. Our short $15 \mathrm{~min}$ field test (as displayed on Fig. 2) suggested a dampening of the gauge-recorded signal for the first $3 \mathrm{~min}$ initial phase of the generated hyetograph and the slightly longer 5 min broadening at the final phase of hyetograph. Detailed discussion of the origins of gauge step response errors is beyond the scope of this paper and is in fact hard to realize since it is introduced by the gauge's inner microprocessor algorithm for data processing. This algorithm is known only by the gauge manufacturer and is not reported in the technical documentation.
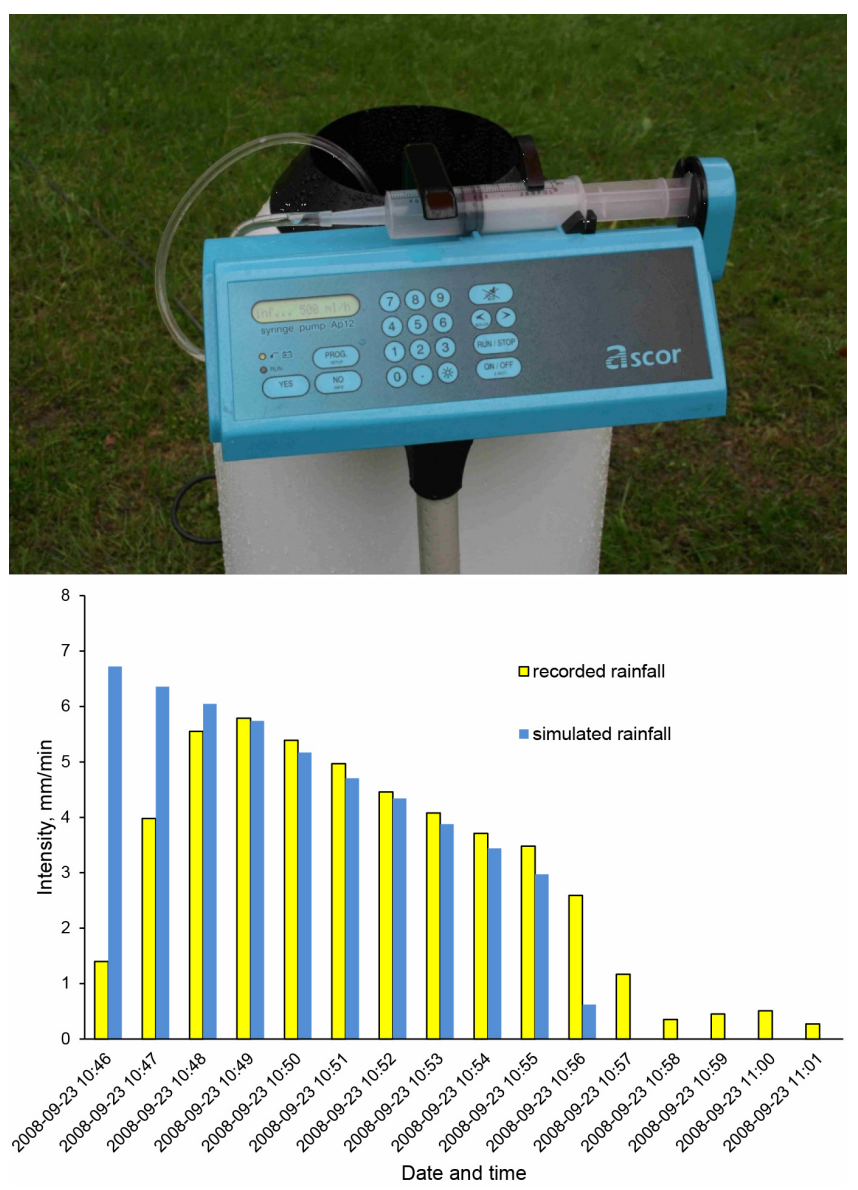

Figure 2. Weighing-type TRwS 200E gauge during some tests (upper panel). Rainfall is simulated by means of a precise medical pump. Sample of test results reporting simulated and recorded rainfall depths (lower panel).

In general, it could only be stated that in weighing-type electronic gauges, the weight of deposed precipitation was sampled by some electronic (often piezometric) sensor with some high temporal resolution at presumably $\mathrm{kHz}$ rate. Afterwards all samples were averaged over longer time windows, unknown to the user. This process was repeated for overlapping time windows, and the difference of the rainfall total of adjacent windows was calculated to obtain the temporal rainfall rate reported as instrument output at its recording time resolution. In addition, rainfall rates were always rounded regardless of the magnitude of real precipitation (resulting in additional rounding errors discussed afterwards). This procedure allowed for satisfactory smoothing of electronic sensor signal fluctuation due to wind effects and temperature changes. It allows for the introduction of some additional filters, cutting sudden signal jumps due to foreign-object deposition inside the open orifice of the gauge inner tank (e.g., falling leaves or acts of vandalism by throwing small stones or garbage). 


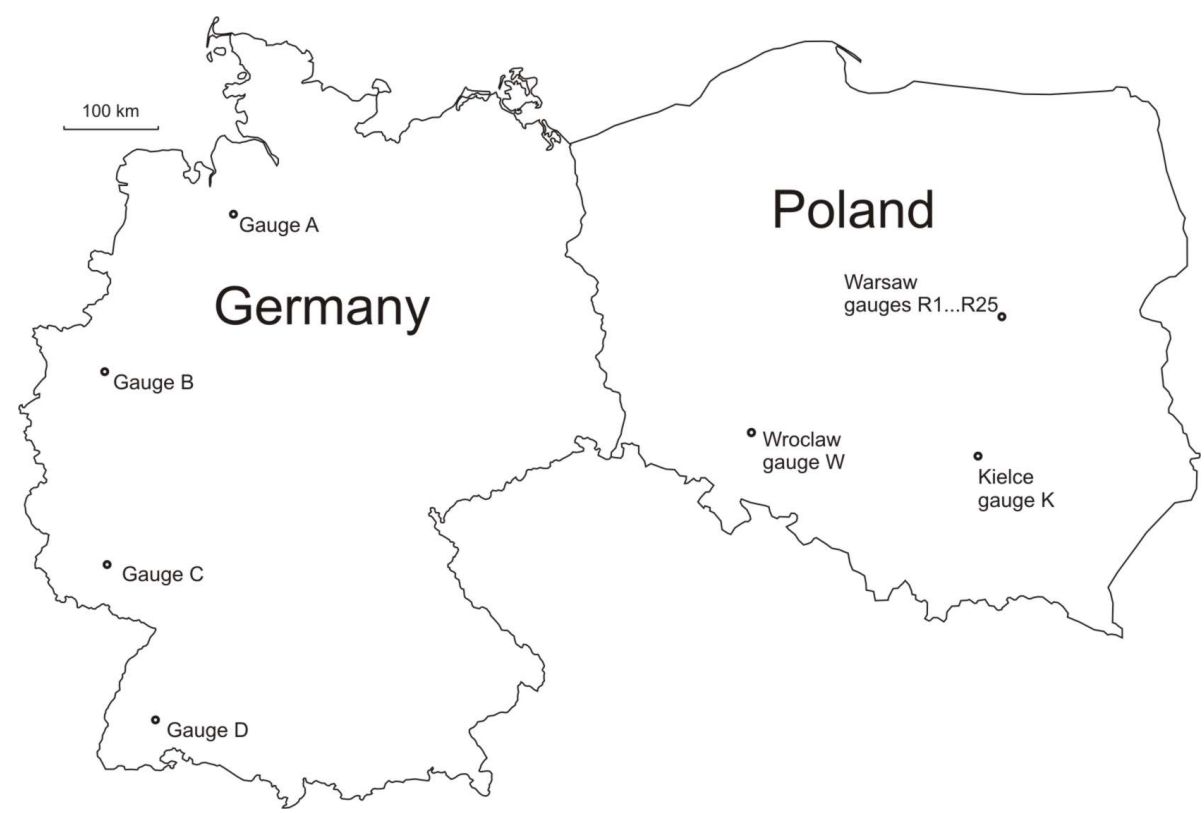

Figure 3. Location of Polish and German precipitation gauges used during the comparison of Warsaw results with other studies.

In light of our personal experiences and test results of the WMO (Lanza et al., 2005), it could be stated that reliable precipitation recording at single minute scales by commercially available gauges is still the goal to be achieved and not a current reality. Having this in mind, as well as timescales of previous microcanonical cascade studies concerning urban hydrology realized on time series recorded by old-type gauges, we decided to work with the aggregated precipitation time series at $5 \mathrm{~min}$ resolution. The technique used to aggregate original $1 \mathrm{~min}$ data into $5 \mathrm{~min}$ time series is discussed afterwards; here we only mention that this operation was opposite to the rainfall total differentiation for adjacent time windows operated by the gauge microprocessor.

Despite the limited timespan of available data covering the period from the 38th week of 2008 to the 49th week of 2010, we believe that the Warsaw precipitation network might support good probing ground for the variability study in the microcanonical cascade parameters over small-scale urban areas. In fact, the Warsaw precipitation-monitoring network belongs to the biggest European urban gauge network. Its size can be compared only with similar networks of 25 gauges in Vienna $\left(414.87 \mathrm{~km}^{2}\right)$ or with 24 gauges spread throughout Marseille $\left(240.62 \mathrm{~km}^{2}\right)$ and Barcelona $\left(100.4 \mathrm{~km}^{2}\right.$ ) (see TTT, 2010).

We compare the results of our study with those related to other Polish and German gauges. We limit our comparison to results previously published by Licznar et al. (2011a, b) for four gauges in Germany (gauges A, B, C and D represent local climates of different parts of western Germany) and one gauge in Wroclaw, Poland and yet unpublished results by Górski (2013) for a rain gauge in Kielce, Poland (Fig. 3). Our choice is motivated by the similarity of the used methodology and the investigated range of timescales, as well as by the indispensable accessibility to precise recordings of the BDC histograms.

Finally, to investigate the existence of possible statistical bias induced by the calculation of BDCs on short precipitation records, we use additional data recorded by an oldtype pluviograph gauge installed previously at the current location of gauge R7 on the ground of Lindley's Filters station. This pluviograph gauge was operated only in summer months from 1 May to 31 October. Data are in the form of 15 min rainfall time series read off the original paper strips with the resolution of $0.1 \mathrm{~mm}$ for depth, covering a period of 25 years (1983-2007).

\subsection{Microcanonical cascade models}

We use MCMs as in Licznar et al. (2011a, b). We consider the disaggregation of precipitation totals from $1280 \mathrm{~min}$ (quasi daily) into $5 \mathrm{~min}$ time series, assuming the branching number $\mathrm{b}$ equal to 2 , and constructing cascades assembled from only nine levels $(n=8, \ldots, 1,0)$ corresponding to timescales $\lambda=2^{n}$ from $\lambda=256$ to $\lambda=1$ (Fig. 4). Precipitation-depth time series generated by such cascades are the products of the original precipitation total $R_{0}$ at timescale $\lambda=256$, multiplied by the sequence of weights at the descending cascade levels:

$R_{j, k}=R_{0} \prod_{i=1}^{k} W_{f(i, j), i}$,

where $j=1,2, \ldots 2^{k}-1,2^{k}$ marks the position in the time series at the $k$ th cascade step. The sequence of randomly 


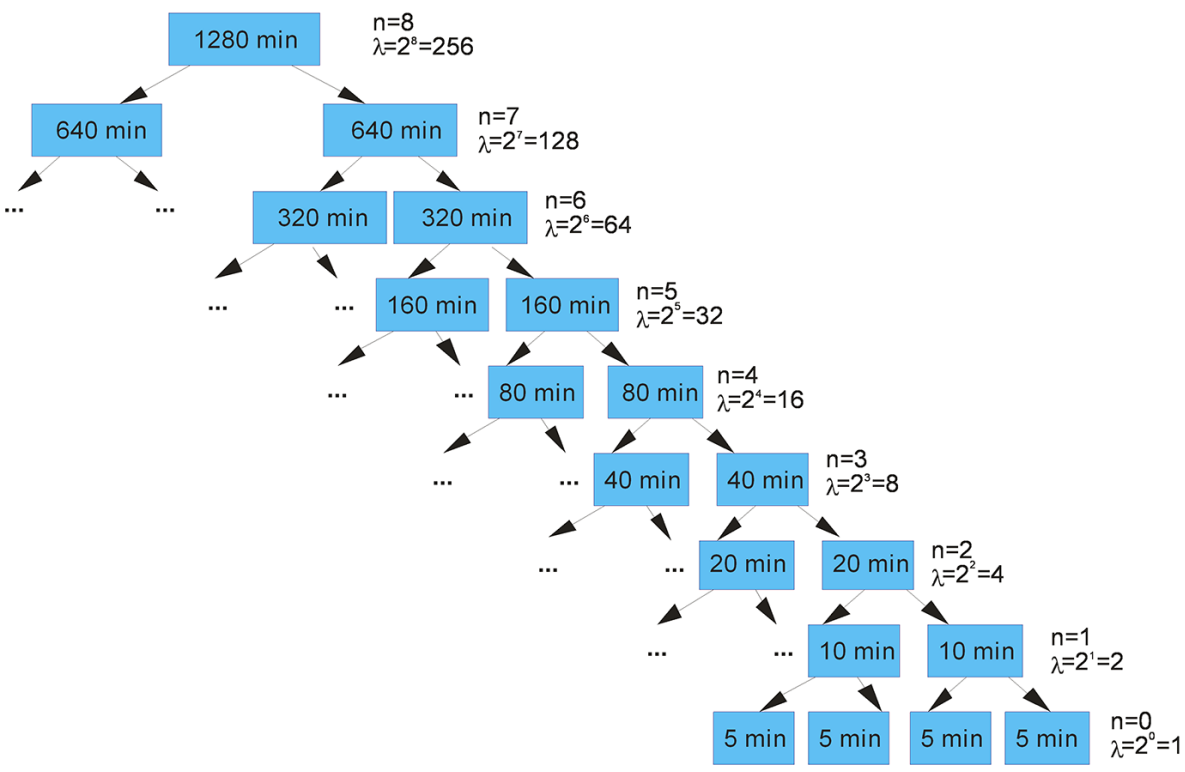

Figure 4. Schematic diagram of a developed microcanonical cascade model with branching number $b=2$.

generated weights $W_{f(i, j), i}$ is steered at the following $i$ th cascade step by the function $f(i, j)$, which rounds up $j / 2^{k-i}$ to the closest integer. The weights in the microcanonical cascades are forced to sum to 1 so their pairs are always equal to $W$ and $1-W$, where $W$ is a two-sided truncated random variable from 0 to 1 . The microcanonical assumption conserves the mass (precipitation depth in our case) at each branch and eliminates the risk of cascade degeneration. From an engineering perspective, this means that the downscaling process can be seen as opposite to precipitation summation realized by Hellman gauges, recording daily totals only, and a pragmatic solution for the generation of synthetic precipitation time series at $5 \mathrm{~min}$ resolution.

In our study we do not focus our attention on the disaggregation capabilities of microcanonical cascades already discussed in numerous papers. We concentrate on the smallscale variability of their generators $W$ among gauges constituting the urban precipitation network. The obvious attractiveness of MCMs arises from the possibility of extracting the distribution of $W$ from data on the basis of BDC studies (Cârsteanu and Foufoula-Georgiou, 1996). By definition, BDCs are generally calculated using non-overlapping adjacent pairs of precipitation time series:

$\mathrm{BDC}_{j, \tau}=\frac{R_{j, \tau}}{R_{j, \tau}+R_{j+1, \tau}} \quad j=1,3,5, \ldots, N_{\tau}-1$,

where $R_{j, \tau}$ is the precipitation amount for the time interval of length $\tau$ at position $j$ in the time series, and $N_{\tau}$ is the length of time series at timescale $\tau$. The calculation of BDCs with respect to Eq. (2) for Warsaw gauges is conducted only for non-zero pairs of $R_{j}$ and $R_{j+1}$. Calculations are executed at aggregated intervals of length $2^{n} \tau_{\text {org }}$, where $\tau_{\text {org }}$ is the original time step equal to 5 min and $n$ is a cascade level, increasing from 0 to 8 with increasing cascade timescales $\lambda$ from 1 to 256 (Fig. 4). Simultaneously for all analyzed timescales, BDC couples equal to $0 / 1$ or $1 / 0$ (when only one between $R_{j}$ and $R_{j+1}$ is 0 ) are separated from resulting data sets and their occurrence probabilities, $p_{0}$ (LEFT) and $p_{0}$ (RIGHT), respectively, are used to estimate intermittency probability $p_{0}$ :

$$
\begin{aligned}
& \operatorname{Pr}\left(\mathrm{BDC}_{n}(j)=0 \text { or } \mathrm{BDC}_{n}(j+1)=0\right) \\
& =p_{0}(\mathrm{LEFT})+p_{0}(\mathrm{RIGHT})=p_{0} .
\end{aligned}
$$

The probability $p_{0}$ is used within a MCM generator to take into account the intermittency characteristic of precipitation, forcing some portion of random weights $W$ to be equal to 0 .

The preliminary results have revealed an overrepresentation of BDC values equal to $1 / 2$ or $1 / 3,2 / 5$ and $1 / 4(2 / 3,3 / 5$ and $3 / 4$, respectively), especially for small timescales, i.e., $\lambda=1$ and $\lambda=2$. Figure 5 (left panel) shows an example of BDC histogram for timescale $\lambda=1$ with evident artificial spikes. Similar phenomenon was already reported by Rupp et al. (2009) and Licznar et al. (2011b) and explained as the result of the instrument or the recording precision of precipitation gauges. The magnitude of observed rounding errors for Warsaw gauges is, however, smaller than in the case of German gauges (Licznar et al., 2011b); the precipitation depths recorded with a better resolution of $0.001 \mathrm{~mm}$ still result in irregularity of BDC distribution, induced by sharp peaks at discrete BDC values, and hinder the identification of the theoretical distribution. In order to correct the rounding errors, a randomization procedure originally proposed by Licznar et al. (2011b) is applied. This type of procedure, also known as jittering, 

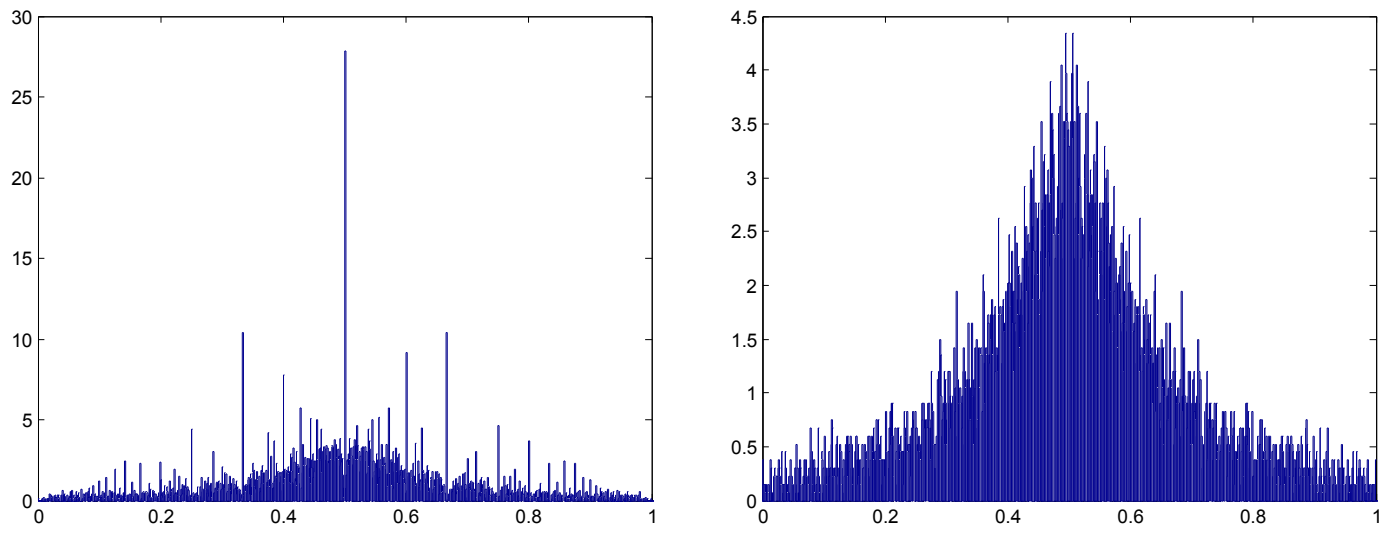

Figure 5. Comparison of BDC histograms for gauge R7 and timescale $\lambda=1$, calculated according to the non-overlapping moving window algorithm and using original (left panel) and randomized (right panel) non-zero precipitation data. Horizontal axes show BDC range and vertical axes show the respective frequency values.

is fundamental to the analysis of data characterized by the presence of ties (De Michele et al., 2013). Thus, the original 1 min time series are slightly modified by adding some random corrections to the precipitation depths exceeding zero. Random correction values are sampled from the uniform distribution in the range $[-0.0005,0.0005] \mathrm{mm}$, resulting in visible BDC histogram smoothing (Fig. 5, right panel). Note that the uniform distribution is used for the randomization of the rounding errors because, in the absence of information, it is the most intuitive distribution requiring less assumption (for more details, please see Licznar et al., 2011b).

Irregularities in BDC histograms are observed for timescales $\lambda>8$. These are due to the decreasing sample size, calculated from a limited timespan of accessible data slightly exceeding 2 years. This issue is rather irrelevant in former studies (Molnar and Burlando 2005, 2008; Licznar et al., 2011a, b) realized on data series 10 or even 20 times longer. To solve this issue, we apply the overlapping moving window algorithm as an alternative to the classical nonoverlapping moving window algorithm for the calculation of BDC values. Figure 6 shows the differences between the two algorithms for $\lambda=1$. Switching from non-overlapping to overlapping moving window algorithm leads to an increase of the number of time segments for the calculation of BDC values. For time series of $n$ data and a time window of size $m \leq n$, the number of non-overlapping windows is $\lfloor n / m\rfloor$, where the symbol $\lfloor\cdot\rfloor$ represents the integer part while the number of overlapping windows is $(n-m+1)$. For large $n \gg m$, the overlapping moving window algorithm leads to almost $m$ times the number of time segments available in the overlapping moving window algorithm. It should be stressed that the real strength of the overlapping moving window algorithm in analyzing distributions of BDC values can be observed for the largest timescales. The reason is that for small timescales most of the time segments are characterized by zero precipitation and thus not involved in the calculation of BDCs, whereas for larger timescales, time segments are becoming larger and rarely characterized by zero precipitation. This phenomenon arises from the fractal properties of rainfall time series, and similar conclusions result from the "box-counting" analysis.

It is clear that the overlapping moving window algorithm is especially desired for limited observational data sets. However, its implementation for short time series may be characterized by a poor representativeness of BDC distributions due to multi-decadal oscillations of precipitation totals and extremes (Willems, 2013). To investigate the magnitude of the oscillations in the BDC distributions, we use historical time series from former old-type gauge R7 covering a 25-year period from 1983 to 2007 at $15 \mathrm{~min}$ resolution. For each year, there are only 6 months of data from May to October available. For this data set, we make the calculations of BDCs in seven time periods. First, we calculate BDCs for the 5-year periods 1983-1987, 1988-1992, 1993-1997, 1998-2002 and 2003-2007 using the overlapping moving window algorithm. We consider this temporal size $(5$ years $\times 6$ months $=30$ months) because it is comparable to the one available for electronic gauges. Afterwards, we repeat the same calculation with a 25-year size using both non-overlapping and overlapping moving window algorithms. As we work here with a coarser resolution $(15 \mathrm{~min}$ instead of $5 \mathrm{~min}$ of electronic gauges), we perform the analysis with a smaller hierarchy of sub-daily timescales $\lambda^{\prime}$ from 1 to 32 and breakdown times from 15 to $30 \mathrm{~min}$ up to 480 to $960 \mathrm{~min}$. For all calculations we perform the randomization of non-zero values. Since their reading precision was set to $0.1 \mathrm{~mm}$, we introduce a random correction belonging to the uniform distribution in the range of $[-0.05,0.05] \mathrm{mm}$.

To compare BDC histograms obtained for all analyzed timescales $\lambda$ and $\lambda$, with theoretical functions, a probability distribution assembling two truncated (with truncation points at 0 and 1) $\mathrm{N}$ distributions (Robert, 1995) and one $\mathrm{B}$ 
1-min precipitation time series
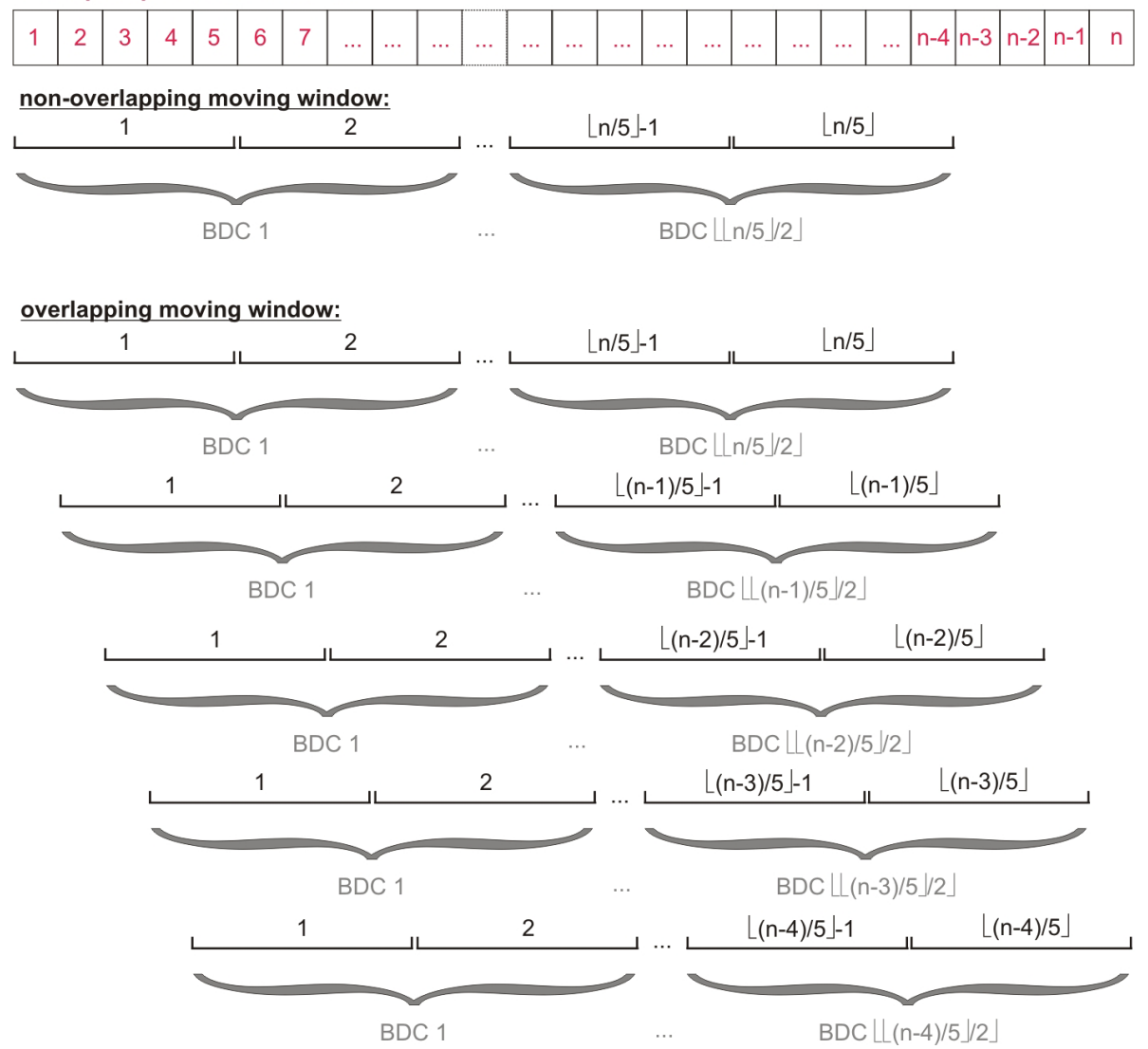

Figure 6. Example showing differences between non-overlapping and overlapping moving window algorithms for the calculation of BDCs in the case of $1 \mathrm{~min}$ precipitation time series and breakdown time of 5-10 min. Note that $\lfloor n\rfloor$ means the integer part of $n$, where $n$ is the total length of 1 min precipitation time series.

symmetrical distribution is implemented. This distribution, indicated as $2 \mathrm{~N}-\mathrm{B}$ distribution, has the following density function:

$$
\begin{aligned}
& p(w)=p_{1}\left\{\frac{1}{\sigma_{1} \sqrt{2 \pi}} e^{\frac{-(w-0.5)^{2}}{2 \sigma_{1}^{2}}}\right\} \\
& +\left(1-p_{1}\right)\left\{p_{2}\left\{\frac{1}{B(a)} w^{a-1}(1-w)^{a-1}\right\}\right. \\
& \left.+\left(1-p_{2}\right)\left\{\frac{1}{\sigma_{2} \sqrt{2 \pi}} e^{\frac{-(w-0.5)^{2}}{2 \sigma_{2}^{2}}}\right\}\right\}
\end{aligned}
$$

where $p_{1}$ and $p_{2}$ are weights characterizing the contribution of the individual distributions within the $2 \mathrm{~N}-\mathrm{B}$ distribution, $\sigma_{1}$ and $\sigma_{2}$ are the scale parameters of truncated $\mathrm{N}$ distributions and $B(a)$ is the symmetrical B function parameterized by $a$.

The fitting of $2 \mathrm{~N}-\mathrm{B}$ distribution parameters is performed numerically by means of maximum likelihood estimation. It is very likely that the use of the model given in Eq. (4), governed by five parameters, can suffer from overparameterization in comparison to the most commonly used
B symmetrical distribution with only one parameter. Note that the application of goodness-of-fit tests (namely the Kolmogorov-Smirnov test or $\chi^{2}$ test) at 1 or $5 \%$ levels of significance gives negative results for both $\mathrm{B}$ and $2 \mathrm{~N}-\mathrm{B}$ distributions. This is because the large sample size of empirical BDCs leads to the rejection of the hypothesis, even in the case of very small differences between observed and theoretical distributions, as also pointed out in Licznar et al. (2011a). Here, we use the Akaike information criterion (AIC) as a measure of the relative quality of $2 \mathrm{~N}-\mathrm{B}$ and $\mathrm{B}$ models for given sets of empirical BDCs. AIC is the maximized value of the log-likelihood function (LL) penalized by the number of model parameters $k$ :

$\mathrm{AIC}=2 k-2 \mathrm{LL}$.

The preferred distribution is the one with the minimum value of AIC. 


\subsection{Cluster analysis}

To our knowledge, until now, the variability of MCM generators among a group of gauges was investigated comparing the value of the parameter of B distribution (Molnar and Burlando, 2008). Here, we prefer to compare directly the empirical distribution of BDCs instead of the parameters of the theoretical distribution, which are possibly biased by fitting errors. We encounter the same problems found in the implementation of statistical tests due to the large sample size. For this we use the cluster analysis to compare the shape of BDC histograms among the stations of the monitoring network in Warsaw and with other Polish and German gauges.

In particular, a hierarchical clustering is used. This is a data-mining tool applied to segment data into relatively homogeneous subgroups, or clusters, where the similarity of the records within the cluster is maximized (Larose, 2005). Prior to the application of the cluster analysis for each timescale and each site, the BDC histogram is sampled in 100 points selected at equal distance one from each other. These 100 values are the components of a vector representing the empirical BDC distribution. Note that a basic requirement of cluster analysis is the comparison of records of equal length. As all $\mathrm{BDC}$ distributions are left and right truncated in the interval $(0,1)$, sampling their histograms with a resolution of 0.01 produces vectors that describe well the shape of histograms. The clustering of these vectors (searching similar sites) is operated using the Euclidean distance. It is computed as

$d_{\text {Euclidean }}(\boldsymbol{X}, \boldsymbol{Y})=\sqrt{\sum_{i}\left(x_{i}-y_{i}\right)^{2}}$,

where $x_{i}$ and $y_{i}$ with $i=1, \ldots, 100$ represent the $i$ th component of $\boldsymbol{X}$ and $\boldsymbol{Y}$ vectors, respectively.

The Euclidean distance is a measure of similarity, not having, in general, a physical interpretation. Initially, in hierarchical clustering analysis, each vector is considered to be a tiny cluster of its own. Then, in following steps, the two closest clusters are aggregated into a new combined cluster. By replication of this operation, the number of clusters is reduced by one at each step and eventually sites are combined into a single huge cluster. During the agglomerative process, the distance between clusters is determined based on single-linkage criterion. In this case, the distance between clusters $\mathrm{A}$ and $\mathrm{B}$ is defined as the minimum distance between any element in cluster A and any element in cluster B. This single linkage is often termed the nearest-neighbor approach and tends to form long, slender clusters, clearly indicating similarities among clustered elements. As a final result of agglomerative clustering, a treelike cluster structure (named dendrogram) is created.

Dendrograms show similarities as well as dissimilarities between BDC distributions among the considered sites and they are prepared separately for all analyzed timescales.
In addition, the cluster analysis is also applied to the intermittency parameter, in this case comparing vectors of eight components: the $p_{0}$ value for the eight timescales $\lambda=$ $1,2,4,8,16,32,64,128$.

\section{Results and Discussion}

Results are presented relative to gauge R7 for brevity. This station has been selected because of its localization in the strict city center, its installation in perfect meteorological conditions on the ground and the existence of former historical rainfall records. Results for the other gauges are qualitatively similar to those shown for R7.

\subsection{Empirical BDC distributions}

BDC histograms are calculated using the non-overlapping moving window algorithm and plotted in Fig. 7 for gauge R7 and a sequence of analyzed breakdown times. It is clearly visible that, despite the randomization procedure that removes pronounced peaks of histograms at certain specific BDC values, like 0.5 or $1 / 3,2 / 5,1 / 4$ and $2 / 3,3 / 5,3 / 4$, respectively (Fig. 5), the plots remain irregular, especially for timescales exceeding $\lambda=8$, reducing the possibility of identifying the proper theoretical distribution. Visible irregularities of BDC histograms increase with increasing timescales, which is an obvious effect of decreasing data sets and thus decreasing populations of calculated BDC values do not allow the production of histograms of fine bins resolution (referring to the populations). Similarly, Fig. 8 reports the distributions of BDC calculated through the overlapping moving window algorithm. The comparison between Figs. 7 and 8 shows how the change of algorithm from a non-overlapping to an overlapping moving window brings evident smoothing of BDC histograms, occurring not only at larger timescales but also at small timescales. Note that the smoothness of BDC histograms in Fig. 8 is comparable with the quality of BDC histograms showed by Licznar et al. (2011b) for German gauges, derived using non-overlapping moving window algorithm for much longer precipitation time series ranging from 27 to 46 years of continuous records. The introduction of the overlapping moving window algorithm allows for the fitting of MCM parameters with the availability of extremely short time series (i.e., 2 years long) in the case of Warsaw gauges. The overall acceptance of overlapping moving window algorithm implementation, including for short rainfall time series, is discussed in Sect. 3.3.

\subsection{Theoretical BDC distributions and their evolution along timescales}

In Fig. 8 we also report the fitted theoretical distributions ( $2 \mathrm{~N}-\mathrm{B}$ distribution in solid red curves and $\mathrm{B}$ distribution in blue dashed lines) for each timescale considered. The visual comparison clearly indicates a better fit of $2 \mathrm{~N}-\mathrm{B}$ (or N-B in 

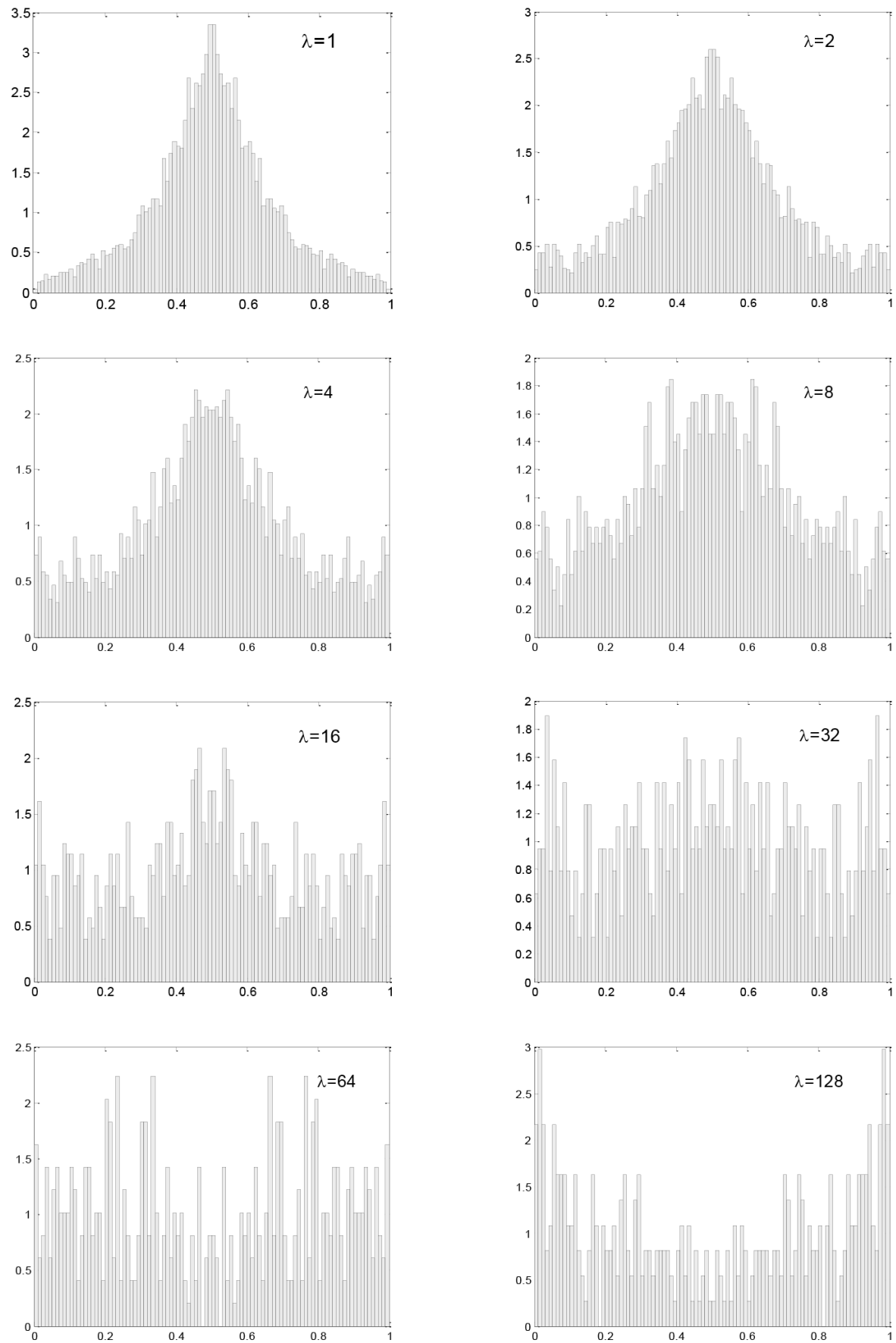

Figure 7. Histograms of BDC values for gauge R7 calculated according to the non-overlapping moving window algorithm and based on randomized precipitation time series. Horizontal axes show BDC range and vertical axes show the respective frequency values.

some cases) distribution for timescales smaller than $\lambda=64$. In Fig. 8 it is possible to see how the distribution with the best fit changes from a $\mathrm{B}$ distribution at $\lambda=128$ to a joined $2 \mathrm{~N}-\mathrm{B}$ for the smallest value of $\lambda$ through a $\mathrm{N}-\mathrm{B}$ distributions. This is in agreement with previous studies by Licznar et al. (2011a, b). This observation is supported by higher values of log-likelihood for $2 \mathrm{~N}-\mathrm{B}$ distribution (or the simplified
$\mathrm{N}-\mathrm{B}$ ) in comparison to the $\mathrm{B}$ distribution (Table 2). These differences are in the range of thousands, and even after accounting for the number of model parameters, the AIC for $2 \mathrm{~N}-\mathrm{B}$ (or the simplified N-B) distributions are much smaller (or equal) than that of $\mathrm{B}$ distributions, confirming the visual result given in Fig. 8. Based on this, we prefer the 2N-B distribution with respect to the $\mathrm{B}$ distribution except for the case 

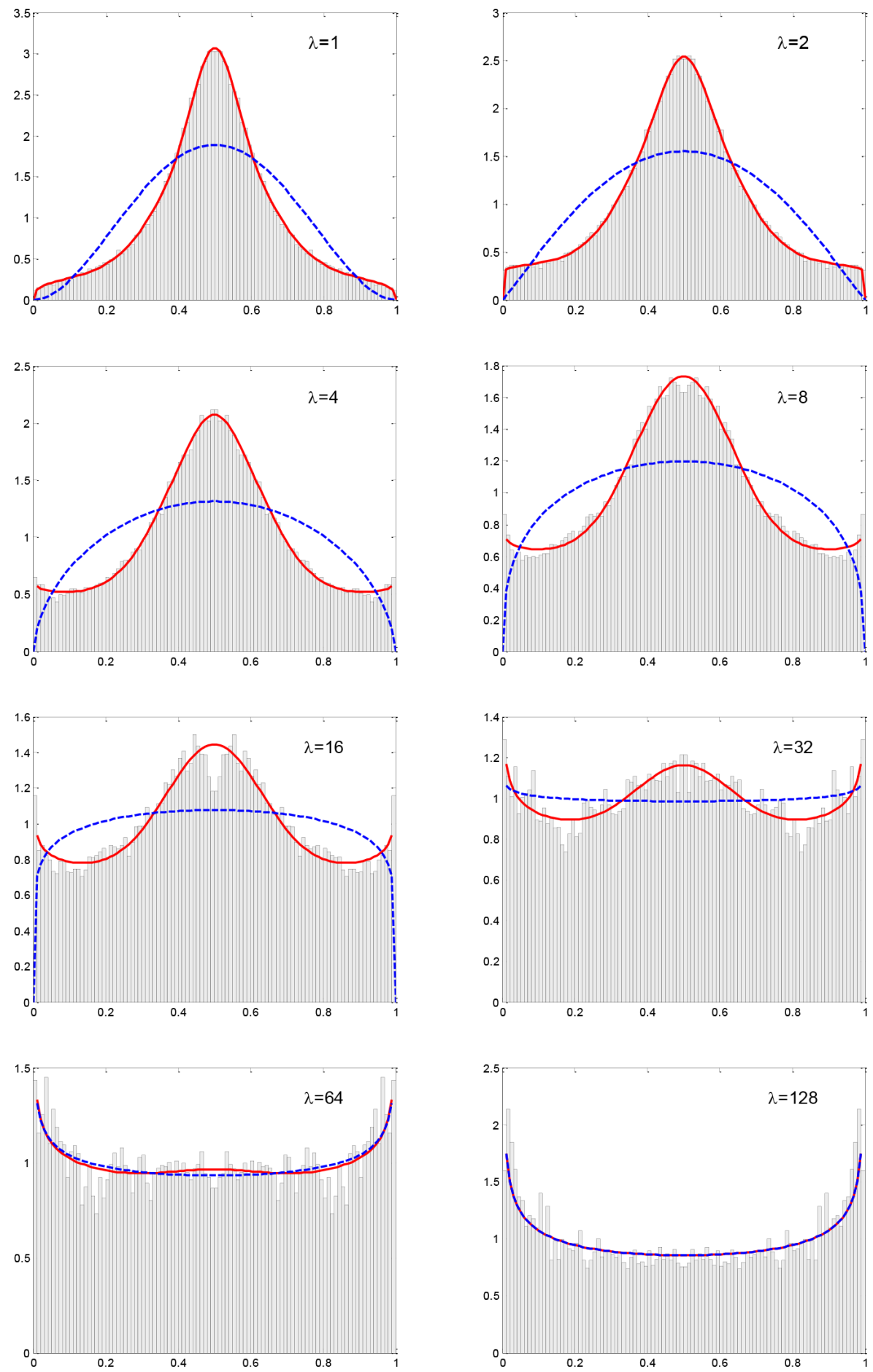

Figure 8. Histograms of BDC values calculated according to overlapping moving window algorithm and based on randomized gauge R7 precipitation times series. Horizontal axes show BDC range and vertical axes show the respective frequency values. The solid red curves represent the $2 \mathrm{~N}-\mathrm{B}$ probability density function, whereas the blue dashed curves represent the B probability density function. 
Table 1. Values of $p_{1}, p_{2}, a, \sigma_{1}$ and $\sigma_{2}$ parameters at different timescales for gauge R7. The values of parameters are reported in bold, whereas their $95 \%$ confidence limits are in italic.

\begin{tabular}{|c|c|c|c|c|c|c|}
\hline $\begin{array}{l}\text { Breakdown } \\
\text { times }\end{array}$ & Timescale & $p_{1}$ & $p_{2}$ & $a$ & $\sigma_{1}$ & $\sigma_{2}$ \\
\hline \multirow{3}{*}{$5-10 \mathrm{~min}$} & \multirow{3}{*}{$\lambda=1$} & 0.1541 & 0.3479 & 1.3350 & 0.0559 & 0.1341 \\
\hline & & 0.1474 & 0.3377 & 1.3097 & 0.0523 & 0.1300 \\
\hline & & 0.1608 & 0.3580 & 1.3604 & 0.0595 & 0.1383 \\
\hline \multirow{3}{*}{$10-20 \mathrm{~min}$} & \multirow{3}{*}{$\lambda=2$} & 0.0706 & 0.4036 & 1.0632 & 0.0559 & 0.1341 \\
\hline & & 0.0644 & 0.3950 & 1.0474 & 0.0523 & 0.1300 \\
\hline & & 0.0768 & 0.4121 & 1.0789 & 0.0595 & 0.1383 \\
\hline \multirow{3}{*}{$20-40 \mathrm{~min}$} & \multirow{3}{*}{$\lambda=4$} & 0.0212 & 0.5036 & 0.9437 & 0.0559 & 0.1341 \\
\hline & & 0.0155 & 0.4954 & 0.9325 & 0.0523 & 0.1300 \\
\hline & & 0.0270 & 0.5118 & 0.9548 & 0.0595 & 0.1383 \\
\hline \multirow{3}{*}{$40-80 \mathrm{~min}$} & \multirow{3}{*}{$\lambda=8$} & - & 0.6175 & 0.9484 & - & 0.1341 \\
\hline & & - & 0.6091 & 0.9390 & - & 0.1300 \\
\hline & & - & 0.6259 & 0.9579 & - & 0.1383 \\
\hline \multirow{3}{*}{$80-160 \mathrm{~min}$} & \multirow{3}{*}{$\lambda=16$} & - & 0.7548 & 0.9170 & - & 0.1341 \\
\hline & & - & 0.7494 & 0.9098 & - & 0.1300 \\
\hline & & - & 0.7601 & 0.9242 & - & 0.1383 \\
\hline \multirow{3}{*}{$160-320 \mathrm{~min}$} & \multirow{3}{*}{$\lambda=32$} & - & 0.8873 & 0.8929 & - & 0.1341 \\
\hline & & - & 0.8827 & 0.8873 & - & 0.1300 \\
\hline & & - & 0.8919 & 0.8985 & - & 0.1383 \\
\hline \multirow{3}{*}{$320-640 \mathrm{~min}$} & \multirow{3}{*}{$\lambda=64$} & - & 0.9797 & 0.8799 & - & 0.1341 \\
\hline & & - & 0.9758 & 0.8754 & - & 0.1300 \\
\hline & & - & 0.9835 & 0.8843 & - & 0.1383 \\
\hline \multirow{3}{*}{$640-1280 \mathrm{~min}$} & \multirow{3}{*}{$\lambda=128$} & - & 1.0000 & 0.7783 & - & 0.1341 \\
\hline & & - & 0.9973 & 0.7754 & - & 0.1300 \\
\hline & & - & 1.0027 & 0.7813 & - & 0.1383 \\
\hline
\end{tabular}

$\lambda=128$. Analyzing the data reported in Table 2 , it is worth noting the systematic increase of sample size $n$ increasing the timescale.

From a practical point of view, a rapid increase in the number of BDCs, equal or close to 0.5, decreasing the timescale should be expected as a symptom of enclosing a limit of the precipitation temporal variability in a point by accessible instruments. The precipitation averaging over some small area of orifice and time intervals is inevitable for gauges; thus, for small timescales, most of the small-scale precipitation variability remains undetected and smoothed, leading to an over-representation of constant precipitation time intervals. From a theoretical point of view, it should be noted that bounded cascades allow the multiplicative weights (or precisely their distributions) to depend on the cascade level and converge to unity as the cascade proceeds. As a consequence, the simulated random process becomes smoother on smaller timescales (Lombardo et al., 2012), which in general mimics the dynamics of precipitation collected by gauges. In other words, as postulated by Marshak et al. (1994), Menabde et al. (1997) and Harris et al. (1998), the variance of weights reduces with every descending cascade level. As a simple extension of this rule, the increasing frequency of weights at the central part of their distribution plots has to be observed. The increase in the number of BDCs equal or close to 0.5 with decreasing timescale is well illustrated by empirical his-
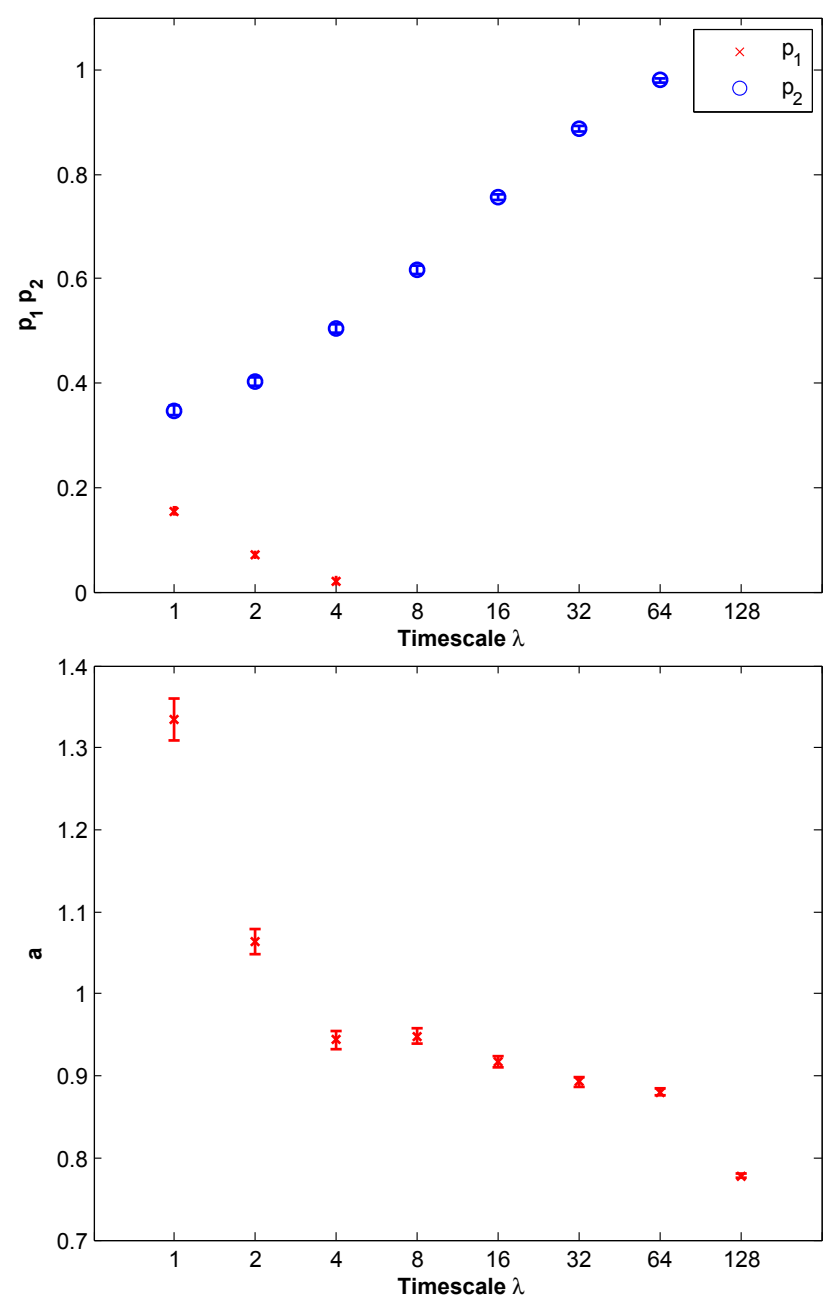

Figure 9. Value and $95 \%$ confidence intervals of parameters of $p_{1}$, $p_{2}$ and $a$ with $\lambda$ for gauge R7. Horizontal axes are plotted at binary logarithm scale $\log _{2}$.

tograms in well-known pioneering contributions to MCM applications for rainfall time series disaggregation published by Olsson (1998), Menabde and Sivapalan (2000) and Güntner et al. (2001). Quite recently, this behavior was also proved to be rainfall-intensity-dependent by Rupp et al. (2009).

For each analyzed timescale, we estimate the parameters of $2 \mathrm{~N}-\mathrm{B}$ probability distribution (or its simplifications $\mathrm{N}-$ $\mathrm{B}$ and B): $p_{1}, p_{2}, a, \sigma_{1}$ and $\sigma_{2}$. Table 1 gives the values for gauge R7 with their $95 \%$ confidence limits. A good visual fit of empirical BDC distributions in Fig. 8 corresponds to quite narrow $95 \%$ confidence limits of the fitted parameters (mostly invisible in Fig. 9 plots). The $95 \%$ confidence limits do not exceed more than a few percent of the estimated values, with the sole exception of parameter $p_{1}$ for $\lambda=4$, where the differences range up to $27 \%$. Additionally, the scale parameters of $\mathrm{N}$ distributions, $\sigma_{1}$ and $\sigma_{2}$, appear to be constant among analyzed timescales not only for gauge R7 but also for the other Warsaw gauges. 
Table 2. Values of the Akaike information criterion (AIC) for the $2 \mathrm{~N}-\mathrm{B}$ distribution (model 1 ) - or its simplifications, N-B and B - and the $\mathrm{B}$ distribution (model 2) and the hierarchy of analyzed timescales $\lambda$ at gauge R7. Calculations were based on estimates of the maximized value of the log-likelihood function (LL), known sample size $(n)$ and number of model parameters $(k)$.

\begin{tabular}{|c|c|c|c|c|c|c|c|c|c|c|c|}
\hline \multirow[b]{2}{*}{$\begin{array}{l}\text { Breakdown } \\
\text { times }\end{array}$} & \multirow[b]{2}{*}{ Timescale } & \multirow[b]{2}{*}{$n$} & \multicolumn{4}{|c|}{ Model 1} & \multicolumn{4}{|c|}{ Model 2} & \multirow[b]{2}{*}{$\begin{aligned} \Delta & =\mathrm{AIC}(\mathrm{M} 2) \\
& -\mathrm{AIC}(\mathrm{M} 1)\end{aligned}$} \\
\hline & & & Distr. & $k$ & LL & AIC(M1) & Distr. & $k$ & LL & $\mathrm{AIC}(\mathrm{M} 2)$ & \\
\hline $5-10 \min$ & $\lambda=1$ & 132940 & $2 \mathrm{~N}-\mathrm{B}$ & 5 & 48480 & -96950 & B & 1 & 36307 & -72612 & 24338 \\
\hline $10-20 \mathrm{~min}$ & $\lambda=2$ & 136968 & $2 \mathrm{~N}-\mathrm{B}$ & 5 & 32272 & -64534 & B & 1 & 19798 & -39593 & 24941 \\
\hline $20-40 \mathrm{~min}$ & $\lambda=4$ & 144778 & $2 \mathrm{~N}-\mathrm{B}$ & 5 & 19071 & -38132 & B & 1 & 8794 & -17585 & 20547 \\
\hline $40-80 \mathrm{~min}$ & $\lambda=8$ & 159272 & N-B & 3 & 11119 & -22232 & B & 1 & 4464 & -8927 & 13305 \\
\hline $80-160 \mathrm{~min}$ & $\lambda=16$ & 185014 & $\mathrm{~N}-\mathrm{B}$ & 3 & 4591.9 & -9178 & B & 1 & 925 & -1848 & 7330 \\
\hline $160-320 \mathrm{~min}$ & $\lambda=32$ & 230716 & N-B & 3 & 1167.3 & -2329 & B & 1 & 46 & -91 & 2238 \\
\hline $320-640 \mathrm{~min}$ & $\lambda=64$ & 315360 & $\mathrm{~N}-\mathrm{B}$ & 3 & 1543.70 & -3081 & B & 1 & 1491 & -2979 & 102 \\
\hline 640-1280 min & $\lambda=128$ & 501092 & B & 1 & 12614.40 & -25227 & B & 1 & 12614 & -25227 & 0 \\
\hline
\end{tabular}

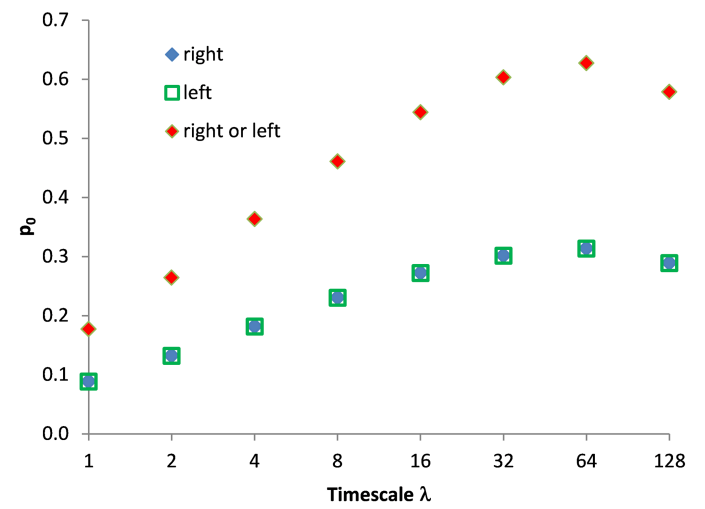

Figure 10. Variability of the intermittency parameter $\mathrm{p}_{0}$ with $\lambda$ for gauge R7. Horizontal axis is plotted at binary logarithm scale $\log _{2}$.

The variability of $p_{1}, p_{2}$ and $a$ with $\lambda$ is presented in Fig. 9 for gauge R7. A systematic decrease of $p_{1}$ down to 0 increasing the timescale is observed, denoting a decreasing importance of the first $\mathrm{N}$ within the $2 \mathrm{~N}-\mathrm{B}$ distribution. An opposite systematic increase of $p_{2}$ up to 1 increasing the timescale is observed, denoting a decreasing importance of the second $\mathrm{N}$ within the $2 \mathrm{~N}-\mathrm{B}$ distribution. The evolution of the B parameter $a$ shows a fast reduction, with values below 1 noticed for the smallest scales, changing the B distribution shape from convex to concave. At larger timescales the reduction of $a$ is hardly visible, with the sole exception of $\lambda=128$. Figure 10 shows the variability of intermittency parameters $p_{0}$ with timescale $\lambda$. For all of them, the values of $p_{0}$ (LEFT) match the values of $p_{0}$ (RIGHT), which is in good agreement with previous studies by Molnar and Burlando (2005) and Licznar et al. (2011a, b). This could be interpreted as the proof of the fully random occurrence of intermittency in the precipitation time series. A systematical increase of $p_{0}$ with $\lambda$ is observed, with the sole exception of a small drop at $\lambda=128$. General increase of $p_{0}$ with timescale is a natural

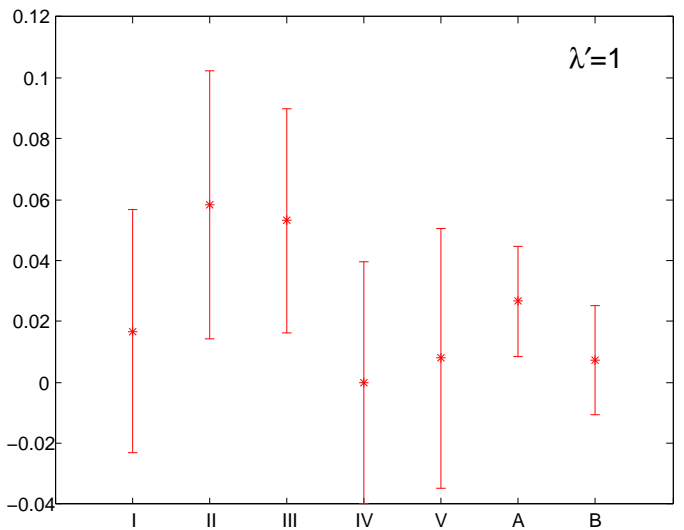

Figure 11. Value and $95 \%$ confidence intervals of parameter $p_{1}$ at timescale $\lambda^{\prime}=1$ for gauge R7. Roman numerals I-V on horizontal axes indicate the 5-year ranges: 1983-1987, 1988-1992, 19931997, 1998-2002 and 2003-2007. Uppercase letters A and B indicate values calculated using the entire 25-year range of 1983-2007 and non-overlapping (A) and overlapping (B) moving window algorithm.

outcome of the fractal properties of the geometric support of rainfall occurrence.

\subsection{Performance of the overlapping moving window algorithm}

The performance of the overlapping moving window algorithm was investigated in detail at gauge $\mathrm{R} 7$, where a 25 -year time series at $15 \mathrm{~min}$ resolution was available. We calculated the parameters of $2 \mathrm{~N}-\mathrm{B}$ distribution for the hierarchy of subdaily timescales $\lambda^{\prime}$ relative to the 5-year periods of 19831987, 1988-1992, 1993-1997, 1998-2002 and 2003-2007 (indicated afterwards with the Roman numerals I, II,..., V, respectively) and the whole 25-year data set (indicated in the next as case A) using the overlapping moving window algorithm. In addition, we calculate the parameters of $2 \mathrm{~N}-\mathrm{B}$ 

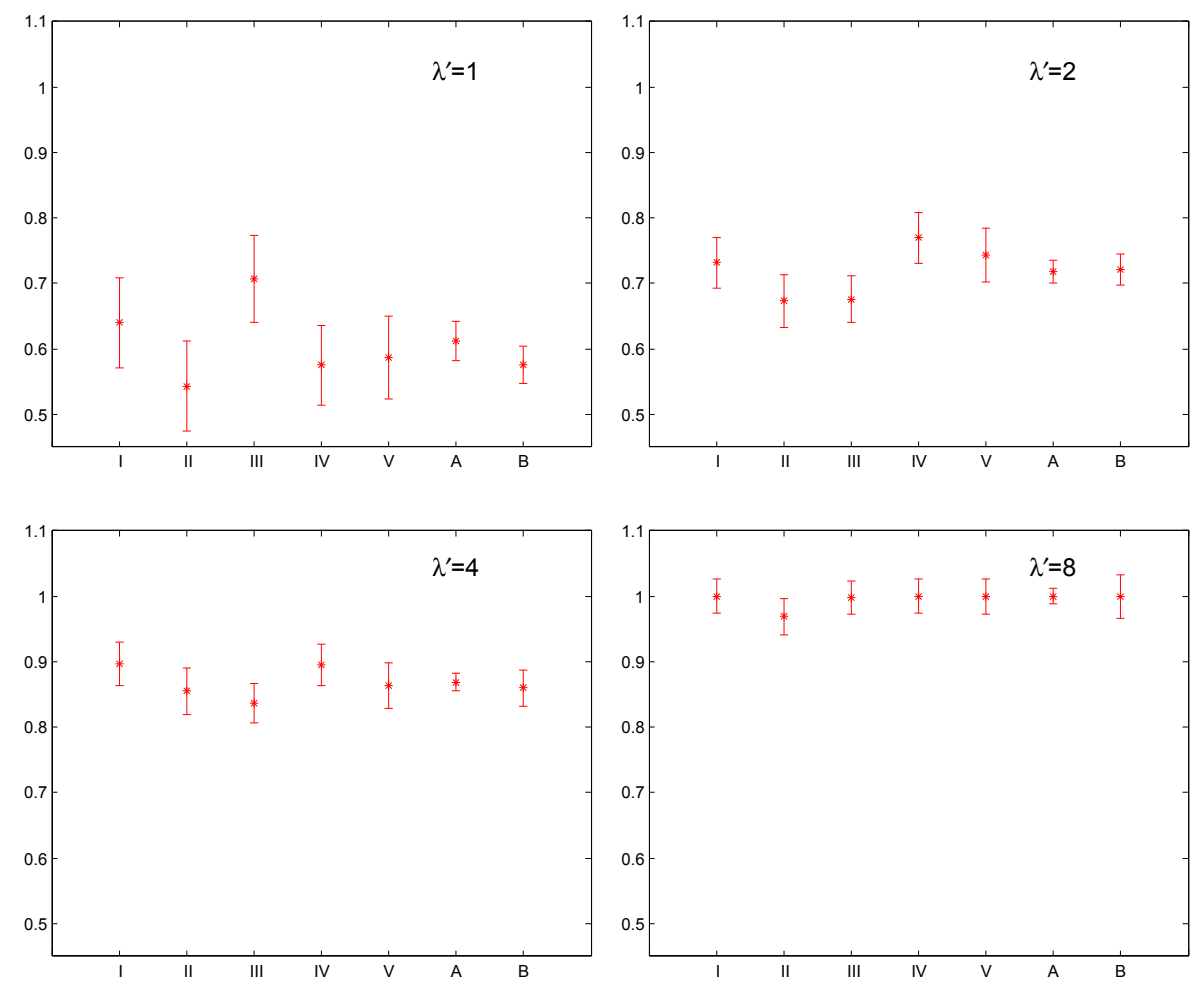

Figure 12. Value and $95 \%$ confidence intervals of parameter $p_{2}$ at timescales $\lambda^{\prime}=1,2,4,8$ for gauge R7. Roman numerals I-V on horizontal axes indicate respectively the 5-year ranges: 1983-1987, 1988-1992, 1993-1997, 1998-2002 and 2003-2007. Uppercase letters A and B indicate values calculated using the entire 25-year range of 1983-2007 and non-overlapping (A) and overlapping (B) moving window algorithm.

distribution also using the classical non-overlapping moving window algorithm over the whole 25 -year data set (indicated in the next as case B). The results are shown in Figs. 11-13.

In general, the selected probability distribution is a B distribution for the largest timescales $\left(\lambda^{\prime}=16,32\right)$, a N-B distribution for $\lambda^{\prime}=2,4,8$ and a $2 \mathrm{~N}-\mathrm{B}$ distribution for $\lambda^{\prime}=$ 1 (the only exception is the period IV). The above listed timescales $\lambda^{\prime}$ are not compatible with timescales $\lambda$; however, transposing them on a coherent time axis leads to the conclusion that characteristic transitions from $\mathrm{B}$ to $\mathrm{N}-\mathrm{B}$ and $2 \mathrm{~N}-\mathrm{B}$ distributions occur at approximately the same time ranges. The estimated parameters $\sigma_{1}$ and $\sigma_{2}$ appear to be constant among analyzed timescales and equal to 0.0646 and 0.1363 , respectively. These values are very close to those reported in Table 1. Figure 11 shows the estimates of $p_{1}$ for $\lambda^{\prime}=1$, with a variability in the range $0-0.058$ for the 5 -year periods I-V. At the same time, the $95 \%$ confidence limits of $p_{1}$ overlap each other partially and values estimated for cases A and B. Confidence limits for periods I-V are rather wide and are only reduced by $50 \%$ for cases A and B. Note that here we work with 15 min time series and not 1 min time series as before.

A better agreement is observed for larger timescales, as illustrated in Figs. 12 and 13, with visibly narrow $95 \%$ confidence limits; however, they still partial overlap one another.
For smaller timescales, larger oscillations of $p_{2}$ parameter can be observed over the periods I-V but, due to wider $95 \%$ confidence limits, they overlap one another and those relative to cases A and B. The only exception is found for the period III at timescale $\lambda^{\prime}=1$.

For parameter $a$ and $\lambda^{\prime}=1,95 \%$ confidence limits for all calculations overlap except period $\mathrm{V}$, which has slightly lower values. For $\lambda^{\prime}=2$ and $\lambda^{\prime}=4$, mutual overlay of $95 \%$ confidence limits is noticed. Passing to $\lambda^{\prime}=8$ and $\lambda^{\prime}=16$, the overlapping among all pairs of periods from $\mathrm{I}$ to $\mathrm{V}$ is not always present; however, it is present with $95 \%$ confidence limits drawn for case B. For $\lambda^{\prime}=32,95 \%$ confidence limits for periods $\mathrm{I}-\mathrm{V}$ and case $\mathrm{A}$ are extremely narrow.

Results reported above suggest good repeatability of BDC distributions calculated during all periods; this is graphically confirmed in Fig. 14, with the exception of period II and timescale $\lambda^{\prime}=1$. Probably this could be explained by the poor performance of the newly proposed overlapping moving window algorithm applied to low time resolution of the original time series. Our observations support the use of the overlapping moving window algorithm for BDC calculations in situations of short (about 2 years) precipitation time series access, while in previous microcanonical cascade studies (e.g., Molnar and Burlando, 2005, 2008), longer (e.g., about $20-30$ years) time series are indispensable. In addi- 

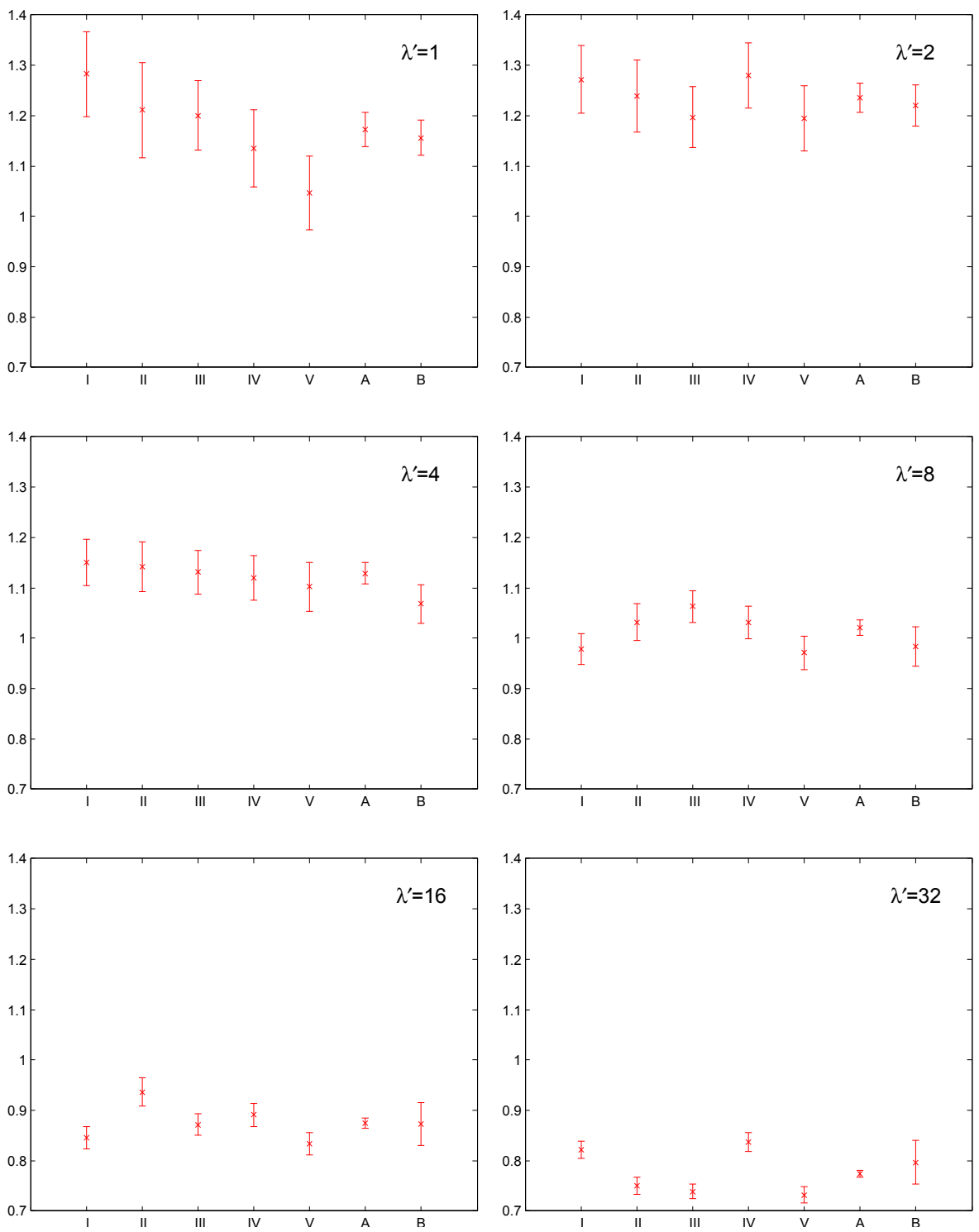

Figure 13. Value and $95 \%$ confidence intervals of parameter $a$ at timescales $\lambda^{\prime}=1,2,4,8,16,32$ for gauge R7. Roman numerals I-IV on horizontal axes indicate the 5-year ranges: 1983-1987, 1988-1992, 1993-1997, 1998-2002 and 2003-2007. Uppercase letters A and B indicate values calculated using all 25-year range of 1983-2007 and non-overlapping (A) and overlapping (B) moving window algorithm.

tion, even in situations of longer precipitation time series access, BDC calculations by means of the proposed algorithm should be favored over the old non-overlapping moving windowtechnique because the new algorithm leads to narrowed $95 \%$ confidence intervals of fitted BDC distributions parameters.

We do not claim here that the moving window technique combined with MCMs solves the problem of local precipitation time series shortage. It is obvious that rainfall statistics derived from short periods may be biased against long-term statistics (e.g., due to climate oscillations). Until now, to our best knowledge, there have been no attempts made to assess the possible bias of MCM generators due to precipitation os- cillations driven by climate change. Hitherto contributions of MCM generators are mostly based on precipitation series that are not too long, presumably displaying only very weak, if any, oscillations and are always treated as a single data set.

Possible bias of MCM generators due to precipitation oscillations undoubtedly should be verified on other, much longer time series of better resolution, such as the $10 \mathrm{~min}$ time series collected in Uccle, Belgium (Willems, 2013). Simultaneously, only a detailed analysis based on long and complete precipitation time series covering at least a few decades could deliver us the answer to the question of whether the climate change effects could be retrieved via the temporal evaluation of microcanonical cascade generators. 

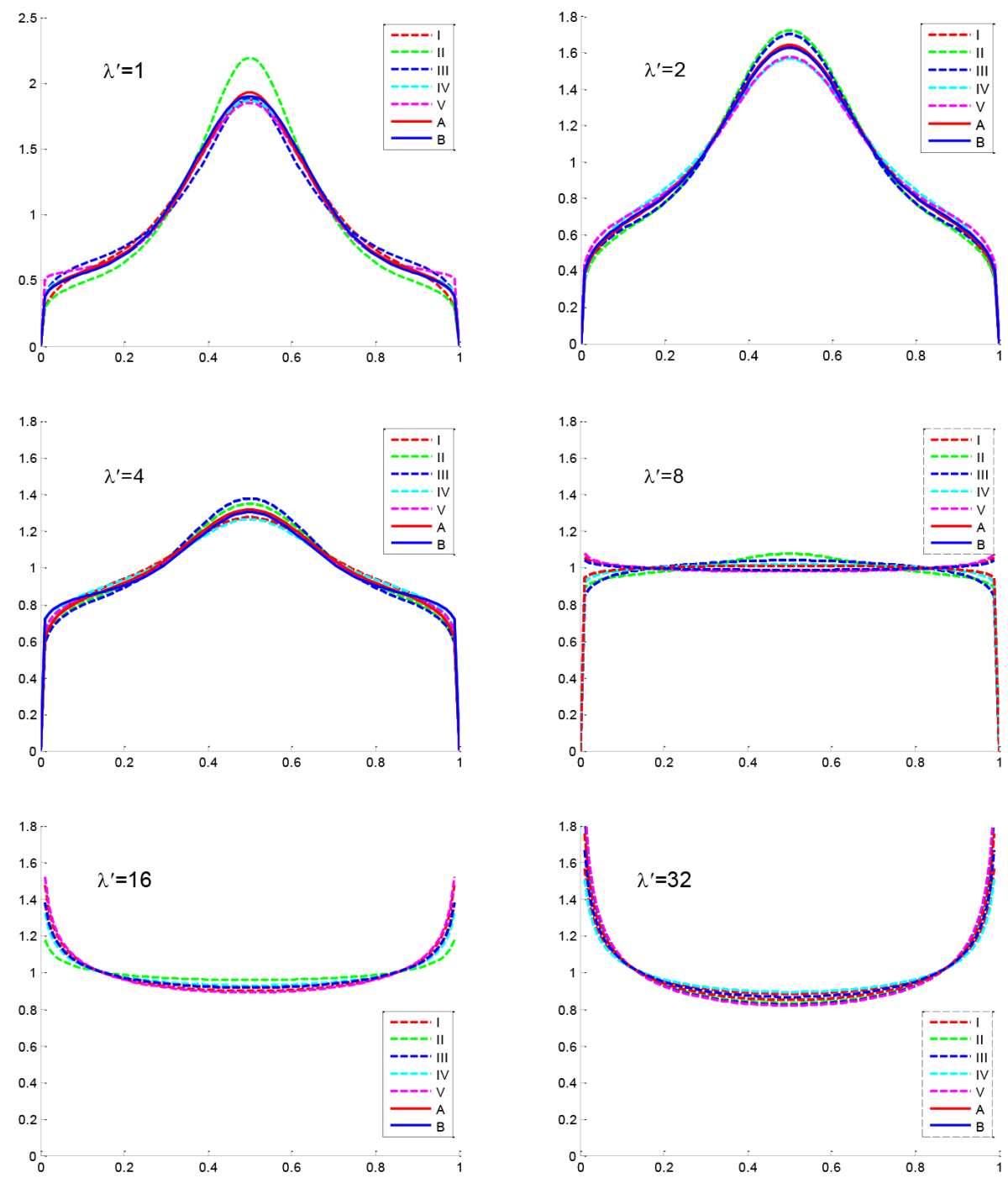

Figure 14. Variability of fitted theoretical BDC distributions histograms at timescales $\lambda^{\prime}=1,2,4,8,16,32$ for gauge R7. Roman numerals IV in legend indicate the 5-year ranges: 1983-1987, 1988-1992, 1993-1997, 1998-2002 and 2003-2007. Uppercase letters A and B indicate results calculated using all 25-year range of 1983-2007 and non-overlapping (A) and overlapping (B) moving window algorithm. In all plots, horizontal axes show BDC ranges and vertical axes show the frequency values.

From this perspective, the moving window technique could be of considerable usefulness in BDC distributions fitting for periods corresponding to 11-year solar spot cycles.

\subsection{Performance of microcanonical cascade in disaggregation}

As an additional check of the overall performance of the applied techniques (i.e., the randomization procedure, the overlapping moving window algorithm and the $2 \mathrm{~N}-\mathrm{B}$ probability distribution), we test the performance of microcanonical cascade in disaggregating the precipitation at the analyzed gauges. The MCM is used to generate 100 synthetic time series at $5 \mathrm{~min}$ resolution on the basis of the observed 1280 min precipitation totals (similar to Molnar and Bur- lando, 2005; Licznar et al., 2011a, b). To evaluate the goodness of disaggregation, we compare the probability of zero precipitation at synthetic and observed time series for all analyzed timescales. Moreover, we calculate the survival probability function of non-zero synthetic precipitation amounts and compare it to the survival probability function of observed precipitation amounts. This analysis is limited to 5 min data, i.e., terminal results of the disaggregation most suitable for urban hydrology application. Special attention to the $5 \mathrm{~min}$ synthetic time series was also paid by other researchers (see e.g., Molnar and Burlando, 2005, 2008; Licznar et al., 2011a, b). An example of $56.3 \mathrm{~mm}$ event disaggregation is plotted in Fig. 15 for gauge R7. It should be stressed that the structure of the synthetic time series is composed 


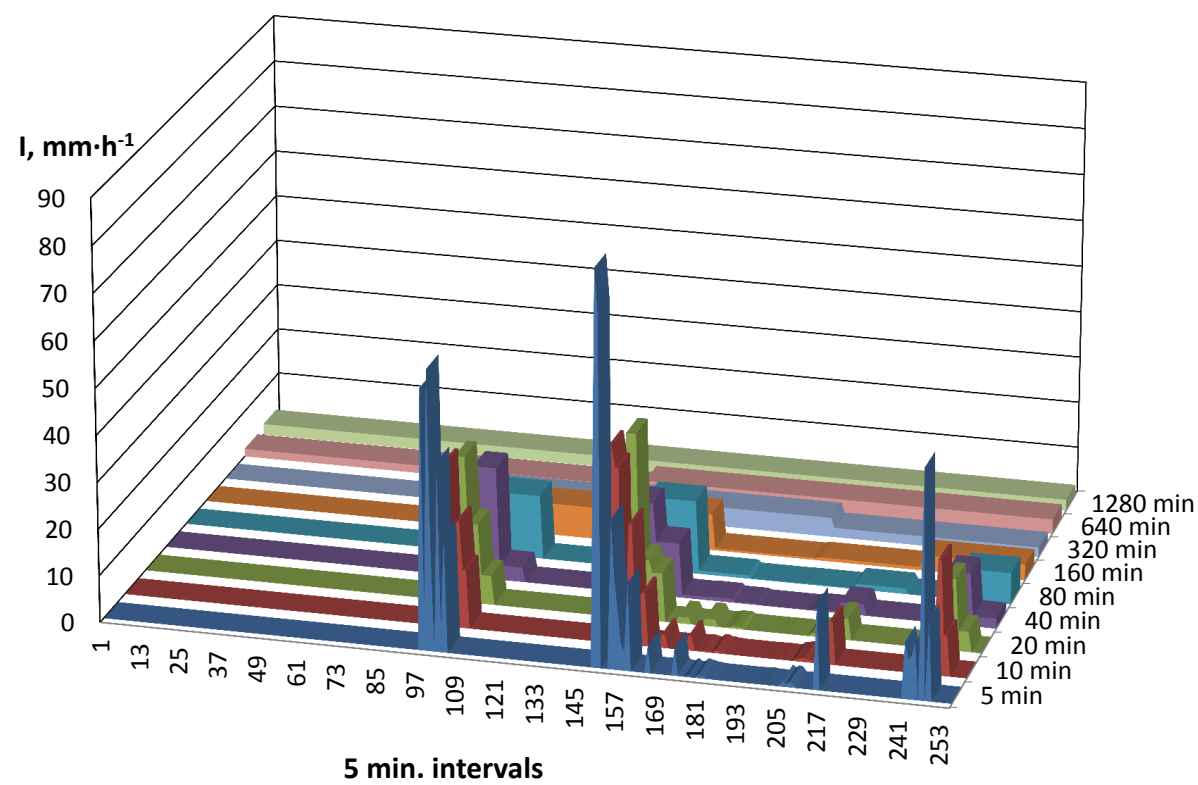

Figure 15. An example of precipitation disaggregation of a $56.3 \mathrm{~mm}$ event from 1280 to $5 \mathrm{~min}$ for gauge R7.

of uncorrelated segments like the one presented in Fig. 15. Thus, the synthetic time series is missing the correct autocorrelation structure of natural precipitation (for detail discussion see Lombardo et al., 2012). The expected value of the zero-precipitation probability $E\left(p_{0}\right)$ for observed and generated series is given in Fig. 16 for gauge R7. The synthetic values of $E\left(p_{0}\right)$ are calculated as the average over $100 \mathrm{MCM}$ disaggregations. The differences in terms of $E\left(p_{0}\right)$ between observed and simulated are negligible (see Fig. 16). In addition, for comparison we also give the synthetic values of $E\left(p_{0}\right)$ for gauges R15 and R25.

Figure 17 shows the comparison between observed and simulated survival probability function of rainfall amount at 5 min for gauge R7. In Fig. 17, for gauge R7, we report the empirical survival probability function for a synthetic series out of 100 and the averaged function using all the generated series. In addition, for comparison, we give the averaged survival functions for gauges R15 and R25. At first glance, highest rainfall intensities drawn in Fig. 17 show strange behavior manifested by constant exceedance probability above a given precipitation threshold. This is especially pronounced for observed or synthetic series from a single MCM run. This is due to the very short rainfall time series used for the calculation of survival probability functions. According to multifractal theory, singularities in a small data set are very rare. Highest rainfall intensities as singularities are very rare in 2-year series. The behavior of both the synthetic functions for gauge R7 in Fig. 17 is very similar, with the sole exception of the extended and smoothed tail of the averaged function plot. Both the synthetic functions are placed above the observed function. This displacement reveals overprediction of $5 \mathrm{~min}$ precipitation depths, particularly at the range of intensities

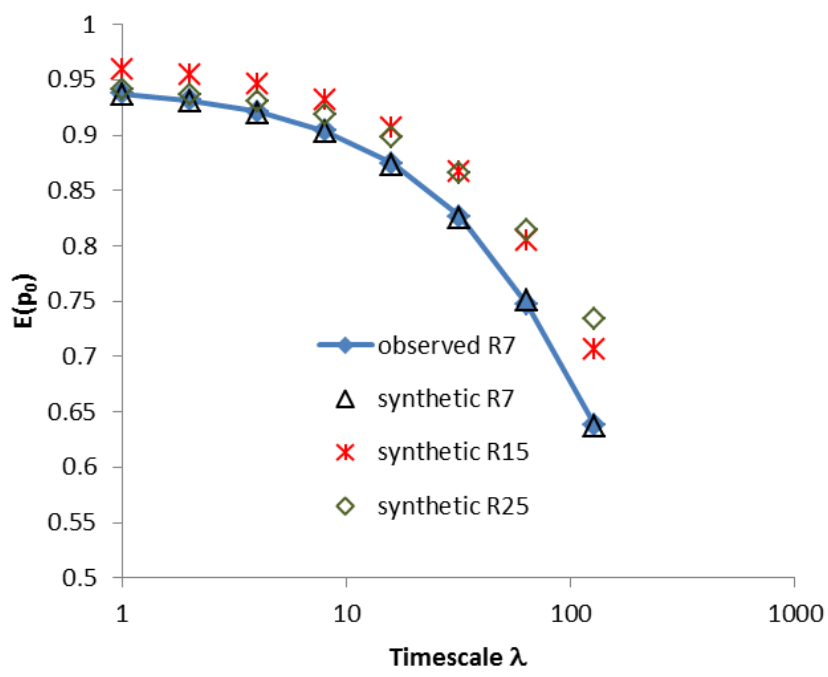

Figure 16. Comparison between observed for gauge R7 and synthetic series for gauges R7, R15 and R25 in terms of intermittency $\mathrm{E}\left(\mathrm{p}_{0}\right)$ for the considered timescales. The values for the generated data are calculated as the average of 100 disaggregation runs. The variability between runs was negligible and thus is not shown here.

from 0.3 to about $2.0 \mathrm{~mm} / 5 \mathrm{~min}$. It should be noted that the magnitude of dissimilarities between synthetic and observed survival functions for gauge R7 did not exceed the ones reported in other works, e.g., Molnar and Burlando (2005) and Licznar et al. (2011a, b). In comparison, the magnitude of dissimilarities between observed survival probability for gauge R7 and synthetic (average) survival probability function for other gauges R15 and R25 is much more pronounced. 


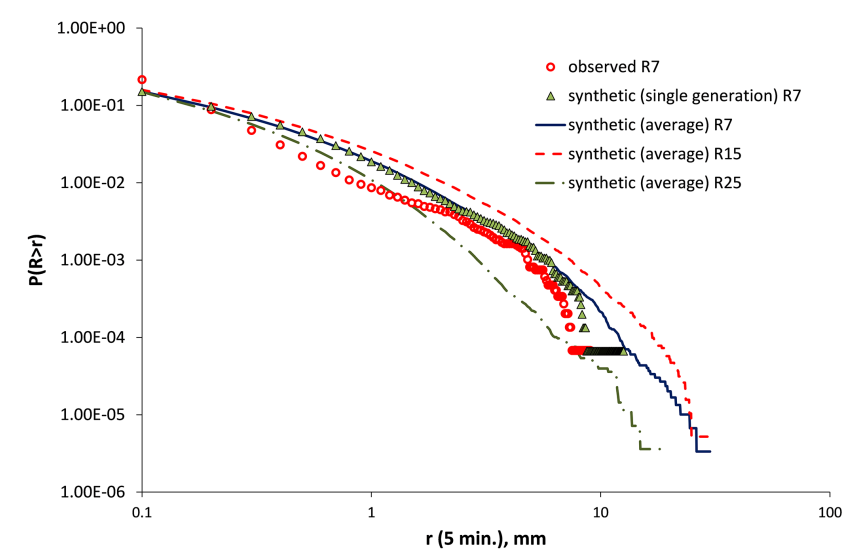

Figure 17. The survival probability function of $5 \mathrm{~min}$ precipitation amounts for the observed time series (circles) and the synthetic time series (triangles) generated by the disaggregation of 1280 precipitation amounts for gauge R7. The lines represent the average distributions calculated over the generation of 100 synthetic time series for gauge R7 and for comparison for gauges R15 and R25.

\subsection{Cluster analysis results and their interpretation}

Dendrograms summarizing the results of the cluster analysis for BDC histograms are produced for each timescale and reported in Figs. 18 and 19 for $\lambda=1$ and $\lambda=128$, respectively. Results for the first four timescales, i.e., $\lambda=1,2,4,8$, are unsurprising and easily interpreted. All Warsaw gauges are grouped in a single cluster with similar shapes of BDC histograms; their interconnection on the dendrogram is placed at the level of binding distance equal to about 0.5. Only R25 seems to be characterized by a slightly different pattern of BDC histogram. However, gauge R25 has a behavior that is still much closer to other Warsaw gauges than to the other cities considered. For example, at $\lambda=1$ gauge R25 is merged into the Warsaw gauges cluster at an Euclidean distance equal to 0.81 , whereas the same occurs for the Kielce (the closest considered Polish city) gauge at the Euclidean distance equal to 1.07. For timescales $\lambda=2,4,8$, gauge R25 merges the cluster of Warsaw gauges at quite similar Euclidean distances: $0.89,0.83$ and 0.81 , respectively.

The dendrogram for $\lambda=128$ is given in Fig. 19, being representative of timescales $\lambda=16,32,64,128$. From Fig. 19 it is possible to see the departure of gauge R15 from the cluster of other Warsaw gauges. The position of gauge R15 is isolated from other Warsaw gauges, and its Euclidean distance from the closest one is large and increases with greater timescale; it is equal to $1.8,3.19,3.88$ and 8.03 for $\lambda=16,32,64$ and 128, respectively. Simultaneously, the Euclidean distance from the cluster of Warsaw gauges to the nearest neighbor does not exceed 0.9, 1, 1.4 and 1.89 for $\lambda=16,32,64$ and 128 , respectively.

This last observation proves that the variability of BDC shapes among Warsaw gauges generally increases with a

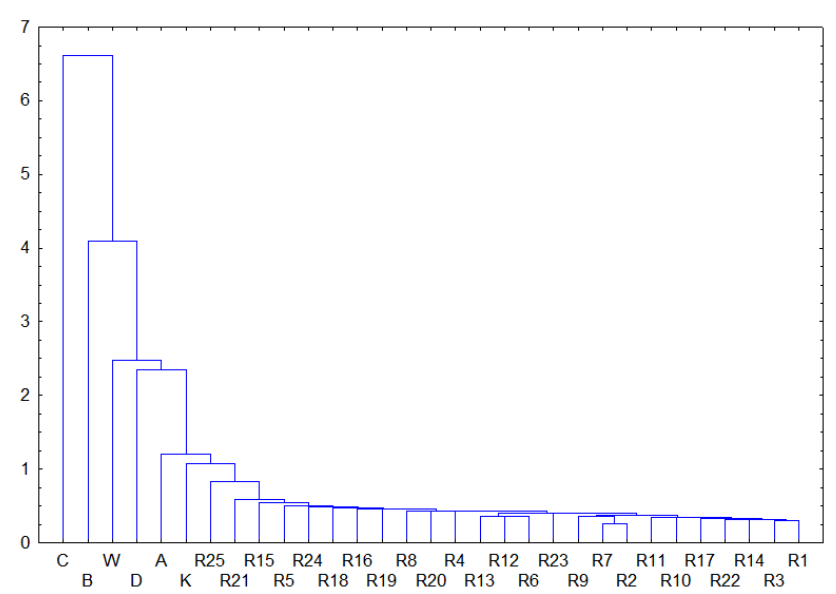

Figure 18. Dendrogram resulting from the cluster analysis of BDC histograms for $\lambda=1$. The vertical scale shows binding distance, whereas names of gauges are given on the horizontal scale ("K" stands for Kielce gauge and "W" stands for Wroclaw).

greater timescale. It may partly be explained by the already mentioned evolution of histogram shapes, the replacement of $2 \mathrm{~N}-\mathrm{B}$ distribution by less centered N-B and Bdistribution characterized by a higher variance of BDC. In the specific case of gauge R15, the BDC histograms for the largest timescales are boldly concave (not shown for brevity) and their shapes are becoming similar to B symmetrical distributions parameterized by very small values of $a$ : $0.76,0.64,0.54$ and 0.45 for $\lambda=16,32,64$ and 128 , respectively.

In the last step we used the cluster analysis to investigate the variability among the gauges in terms of the intermittency parameter $p_{0}$, which is considered a vector with eight components as values corresponding to the considered timescales. Results are given in the form of a dendrogram in Fig. 20. With respect to $p_{0}$, all Warsaw gauges form one single chain-like cluster. Three gauges in the cluster, namely $\mathrm{R} 14, \mathrm{R} 25$ and R15, are characterized by the largest distances from the nearest neighbor with Euclidean distances equal to $0.079,0.064$ and 0.0614 , respectively. The distance of gauges R15 and R25 from the other stations in the cluster is similar to observations made for Figs. 18 and 19. A possible, but not certain, explanation for gauge R14 could be its location close to gauge R15 in a weakly developed part of the city.

Unfortunately, we do not have access to other meteorological data to compare our results with other local climate conditions. To our knowledge, studies about microclimate or local turbulence have not been conducted for Warsaw. However, in our opinion the anomalous behavior of gauges R15 and R25 does not originate from random errors due to gauges installation. As previously mentioned, all gauges were installed in very good conditions, and R15 was an airport gauge. A plausible explanation of the anomalous behavior of gauges R25 and R15 could be found in its location. Gauge R25 was 


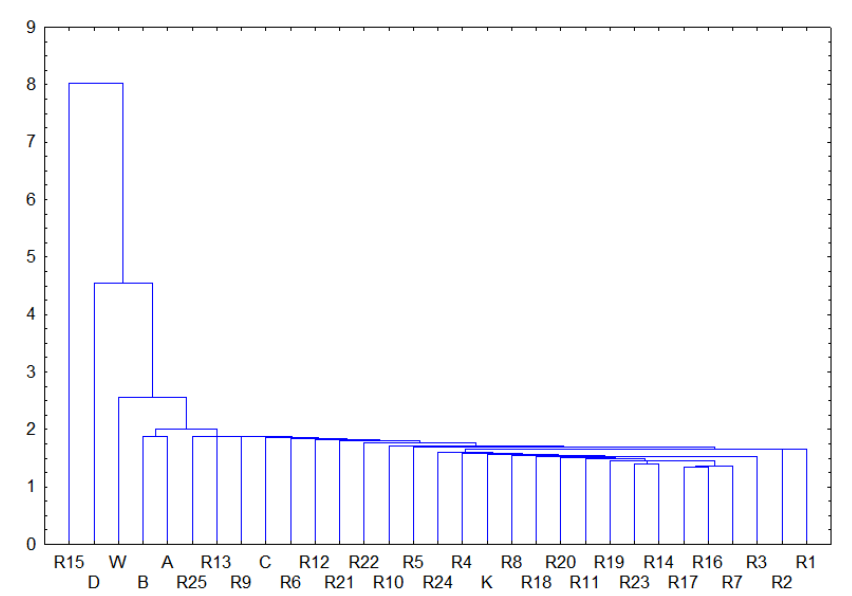

Figure 19. Dendrogram resulting from the cluster analysis of BDC histograms for the timescale $\lambda=128$. The vertical scale shows binding distance, whereas names of gauges are given on the horizontal scale ("K" stands for Kielce gauge and "W" stands for Wroclaw).

located in a southeast suburban area in the close vicinity of a forested area and the Vistula river valley. This specific suburban area is also most frequently a place for the development of local convection processes (Prof. S. Malinowski, personal communication, 2013). The anomalous behavior of gauge R15 seems to arise from its specific location on the ground at the Warsaw airport. In the neighborhood of the instrument there are no high buildings and trees and the ground is covered only by short-cut grass. The local atmospheric turbulence conditions, additionally influenced by taking off and landing aircrafts, could have favored the different behavior of this station. In general, gauges R15 and R25 are the only instruments installed outside the areas of urban fabric (Fig. 1) in rather rural conditions of surrounding green areas. The suburban location of these gauges combined with the direct green surrounding reduces, or even minimalizes to zero, urban heat island effects. Peng et al. (2012) investigated the surface urban heat island intensity across 419 global big cities (including Warsaw). These authors showed that the distribution of daytime surface urban heat island intensity correlates negatively across cities with the difference of vegetation fractional cover and of vegetation activity between urban and suburban areas. Kłysik and Fortuniak (1999) found the occurrence of urban heat in the second biggest city in Poland, Łódź (about $120 \mathrm{~km}$ southwest), which is comparable to Warsaw flat topography. According to statistics calculated over many years at two stations - one in the center and one at the airport - over $80 \%$ of nights were characterized by a surplus heat in town amounting generally to $2-4^{\circ} \mathrm{C}$ and sporadically to $8^{\circ} \mathrm{C}$ and more. Once more for Łódź, Fortuniak et al. (2006) investigated the data from two automatic stations: one urban and one rural. They found the relative humidity to be lower in the town, sometimes by more than $40 \%$, and

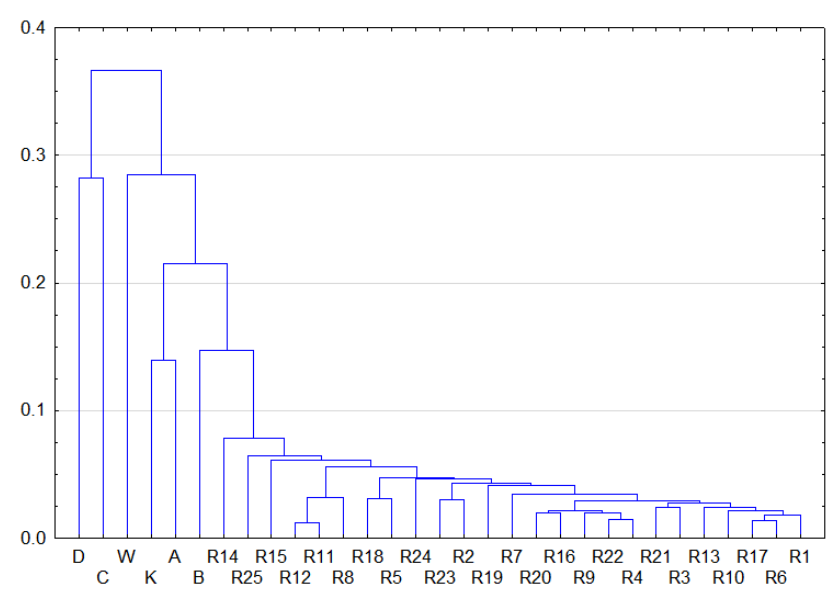

Figure 20. Dendrogram resulting from the cluster analysis of the intermittency parameter $\mathrm{p}_{0}$. The vertical scale shows binding distance, whereas the names of gauges are given on the horizontal scale ("K" stands for Kielce gauge and "W" stands for Wroclaw).

water vapor pressure differences to be either positive (up to $5 \mathrm{hPa}$ ) or negative (up to $-4 \mathrm{hPa}$ ). Air-temperature differences between the urban and rural station exceeded $8{ }^{\circ} \mathrm{C}$. It could be that similar processes occurred in Warsaw and affected local precipitation dynamics and thus gauges R7, R15 and R25. As a consequence, statistics of synthetic time series vary visibly in Figs. 16 and 17. However, the significance of these differences should be studied in more details in the future.

\section{Conclusions}

Keeping in mind the simplicity of the retrieval of microcanonical cascade generators from observational data, we proposed to use this technique for the local variability of very short precipitation time series within an urban monitoring network.

We considered a network of 25 gauges deployed in Warsaw over an area of $517.2 \mathrm{~km}^{2}$. An attempt was made to define the generators of a MCM able to produce 5 min time series, as requested by urban hydrologists, through the disaggregation of quasi-daily precipitation totals. We showed that smooth distributions of BDC are possible for all analyzed timescales, even in the case of a limited length of time series, which in our case slightly exceeded 2 years only. This was made possible by the implementation of a randomization procedure and the use of an overlapping moving window algorithm for the calculation of BDCs.

The correctness of the overlapping moving window algorithm is checked using additional 15 min rainfall time series, 25 years long, at gauge $\mathrm{R} 7$. The algorithm is implemented for a hierarchy of sub-daily timescales and separate 5-year periods. The results of BDC calculations are compared to those obtained using all 25 years of data with both overlapping 
and non-overlapping moving window algorithms. Despite the coarse resolution of data and winter time gaps in the series, the results show a good agreement of BDC distributions calculated over the different periods, suggesting the correctness of the overlapping moving window algorithm, at least in central Poland.

To adequately describe the shapes of BDC histograms, we have implemented a special joined probability distribution, $2 \mathrm{~N}-\mathrm{B}$, assembled from two $\mathrm{N}$ distributions and one $\mathrm{B}$ symmetrical distribution. A systematical evolution of BDC histograms from joined $2 \mathrm{~N}-\mathrm{B}$ through joined $\mathrm{N}-\mathrm{B}$ up to $\mathrm{B}$ distributions was observed increasing the timescale. To test the use of more complicated models alternative to the classical B distribution, we suggested the AIC.

To check all the applied techniques (i.e., the randomization procedure, the overlapping moving window scheme and the $2 \mathrm{~N}-\mathrm{B}$ distribution), MCMs were used to disaggregate $1280 \mathrm{~min}$ precipitation into $5 \mathrm{~min}$ time series. The quality of the generated series was checked, comparing the statistical properties of these with those of observed series. In particular, we compared probabilities of zero precipitation and the survival probability functions of non-zero 5 min precipitation amounts for the considered timescales with agreement comparable to previous studies done in Switzerland, Germany and Poland.

As a main part of this study, we conducted an intercomparison of BDC histograms among the 25 Warsaw gauges and considered as a term of reference another six gauges located in Poland and Germany. The intercomparison was made, scale-by-scale, by means of cluster analysis. Resulting dendrograms for small timescales (i.e., $\lambda=1,2,4,8$ ) revealed rather little variability of BDC histograms among all Warsaw gauges in comparison to the variability exhibited with respect to the other external gauges. Only gauge R25 seemed to be characterized by a slightly different pattern. It might originate from the specific gauge location on the city boundary in the vicinity of forested areas and Vistula river valley.

Dendrograms obtained for large timescales (i.e., $\lambda=$ $16,32,64,128)$ also delivered a general picture of similarity among Warsaw gauges with the very clear exception of gauge R15. To our best knowledge, a possible explanation of this was its installation on the ground at the Warsaw airport, which was strongly man-modified and with turbulent local conditions. In addition, R25, R15 and R14 were also identified as gauges presenting slightly different behavior in terms of the intermittency parameter $p_{0}$.

As final remarks we can affirm that MCMs combined with cluster analysis could be used as a tool for the assessment of the spatial variability of local precipitation patterns among a group of gauges. This framework could be effectively implemented even in the case of very short observational series thanks to the proposed overlapping moving window algorithm. We believe that the use of this algorithm could increase the development and use of MCMs in urban hydrol- ogy. At the same time, we are fully aware of the inherent MCM limitations in the quality of rainfall disaggregation and the necessity of additional verifications of the overlapping moving window algorithm for other gauges with longer and higher quality observational time series.

Returning to questions of interest in urban hydrology addressed at the end of the Introduction, we can formulate following answers:

1. Small precipitation variability within gauges located in city centers, as measured via microcanonical cascade generators, justifies the practice of a single time series use for the probabilistic assessment of the entire urban drainage system.

2. From current engineering needs in urban hydrology, it is enough to use only one fitted MCM for the precipitation time series disaggregation in Warsaw city. We suppose that this result could be valid even in larger urban areas, but the verification is necessary. We dissuade from the cascade generation fitted on precipitation time series collected at instruments located out of the city center in unrepresentative sites like, in our case, the ground at the airport.

3. We question the practice of using gauges from airports for urban hydrology.

Finally, we recommend further research to assess the influence of the local conditions on BDC histograms to find clearer explanations of observed anomalies. We also recognize the necessity of further tests in other cities and precipitation monitoring networks, especially in the case of cities with complicated orography and the presence of hydrological networks.

Acknowledgements. This project was financed by Polish National Science Center (NCN) funds allocated on the basis of decision no. 2011/03/B/ST10/06338. It was realized as a part of the scientific project "Spatiotemporal analysis and modeling of urban precipitation field." Precipitation data were provided by the Municipal Water Supply and Sewerage Company (MWSSC) in Warsaw, Poland. Authors also acknowledge the three anonymous reviewers for their suggestions and comments.

Edited by: R. Uijlenhoet

\section{References}

Ahrens, B.: Rainfall downscaling in an alpine watershed applying a multiresolution approach, J. Geophys. Res., 108, 8388, doi:10.1029/2001JD001485, 2003.

Berne, A., Delrieu, G., Creutin, J. D. and Obled, C.: Temporal and spatial resolution of rainfall measurements required for urban hydrology, J. Hydrol., 299, 166-179, doi:10.1016/j.jhydrol.2004.08.002, 2004. 
BS EN 752-3: Drain and sewer systems outside buildings. Planning, British Standards Institution, ISBN 0580267822, 34 pp., 1997.

Cârsteanu, A. and Foufoula-Georgiou, E.: Assessing dependence among weights in a multiplicative cascade model of temporal rainfall, J. Geophys. Res., 101, 26363-26370, doi:10.1029/96JD01657, 1996.

De Michele, C., Salvadori, G., Vezzoli, R., and Pecora, S.: Multivariate assessment of droughts: Frequency analysis and dynamic return period, Water Resour. Res., 49, 6985-6994, doi:10.1002/wrcr.20551, 2013.

Deutsche Vereinigung für Wasserwirtschaft, Abwasser und Abfall e.V. (DWA): Arbeitsblatt DWA-A 117: Bemessung von Regenrückhalteräumen, Hennef, 2006.

Fortuniak, K., Klysik, K., and Wibig, J.: Urban-rural contrasts of meteorological parameters in Lodz, Theor. Appl. Climatol., 84, 91-101, doi:10.1007/s00704-005-0147-y, 2006.

Gires, A., Onof C., Maksimovic C., Schertzer D., Tchiguirinskaia I., and Simoes N.: Quantifying the impact of small scale unmeasured rainfall variability on urban hydrology through multifractal downscaling: a case study, J. Hydrol., 442-443, 117-128, doi:10.1016/j.jhydrol.2012.04.005, 2012.

Gires, A., Tchiguirinskaia, I., Schertzer, D., and Lovejoy, S.: Multifractal analysis of an urban hydrological model on a Seine-Saint-Denis study case, Urban Water J., 10, 195-208, doi:10.1080/1573062X.2012.716447, 2013.

Górski J.: Analysis of Precipitation Time Series for Needs of Urban Hydrology on Example of Kielce City. PhD thesis, Kielce University of Technology, Poland, 2013 (in Polish).

Güntner, A., Olsson, J., Calver, A., and Gannon, B.: Cascade-based disaggregation of continuous rainfall time series: the influence of climate, Hydrol. Earth Syst. Sci., 5, 145-164, doi:10.5194/hess5-145-2001, 2001.

Harris, D., Menabde, M., Seed, A., and Austin, G.: Breakdown coefficients and scaling properties of rain fields, Nonlin. Processes Geophys., 5, 93-104, doi:10.5194/npg-5-93-1998, 1998.

Hingray, B. and Ben Haha, M.: Statistical performances of various deterministic and stochastic models for rainfall series disaggregation, Atmos. Res., 77, 152-175, 2005.

Kłysik, K. and Fortuniak, K.: Temporal and spatial characteristics of the urban heat island of Łódź, Poland, Atmos. Environ., 33, 3885-3895, 1999.

Koutsoyiannis, D.: Rainfall disaggregation methods: Theory and applications, in: Proc. Workshop on Statistical and Mathematical Methods for Hydrological Analysis, edited by: Piccolo, D. and Ubertini, L., Universitá di Roma "La Sapienza", Rome, http://itia.ntua.gr/en/docinfo/570, 2003.

Lanza, L., Leroy, M., Alexandropoulos, C., Stagi, L., and Wauben, W.: WMO laboratory intercomparison of rainfall intensity gauges, Final report, IOM Report No. 84, WMO/TD No. 1304, 2005.

Larose, D. T.: Discovering knowledge in data: an introduction to data mining, John Wiley \& Sons, Inc., Hoboken, New Jersey, USA, 222 pp., 2005.

Licznar, P.: Stormwater reservoir dimensioning based on synthetic rainfall time series, Ochrona Srodowiska, 35, 27-32, 2013.

Licznar, P., Łomotowski, J., and Rupp, D. E.: Random cascade driven rainfall disaggregation for urban hydrology: An evaluation of six models and a new generator, Atmos. Res., 99, 563578, doi:10.1016/j.atmosres.2010.12.014, 2011a.
Licznar, P., Schmitt, T. G., and Rupp, D. E.: Distributions of microcanonical cascade weights of rainfall at small timescales, Acta Geophysica, 59, 1013-1043, doi:10.2478/s11600-011-0014-4, $2011 b$.

Lombardo, F., Volpi, E., and Koutsoyiannis, D.: Rainfall downscaling in time: theoretical and empirical comparison between multifractal and Hurst-Kolmogorov discrete random cascades, Hydrolog. Sci. J., 57, 1052-1066, doi:10.1080/02626667.2012.695872, 2012.

Marshak, A., Davis, A., Cahalan, R., and Wiscombe, W.: Bounded cascade models as nonstationary multifractals, Phys. Rev. E, 49, 55-69, 1994.

Menabde, M. and Sivapalan, M.: Modeling of rainfall time series and extremes using bounded random cascades and Levy-stable distributions, Water Resour. Res., 36, 3293-3300, doi:10.1029/2000WR900197, 2000.

Menabde, M., Harris, D., Seed, A., Austin, G., and Stow, D.: Multiscaling properties of rainfall and bounded random cascades, Water Resour. Res., 33, 2823-2830, doi:10.1029/97WR02006, 1997.

Molnar, P. and Burlando, P.: Preservation of rainfall properties in stochastic disaggregation by a simple random cascade model, Atmos. Res., 77, 137-151, doi:10.1016/j.atmosres.2004.10.024, 2005.

Molnar, P. and Burlando, P.: Variability in the scale properties of high-resolution precipitation data in the Alpine climate of Switzerland, Wat. Resour. Res., 44, W10404, doi:10.1029/2007WR006142, 2008.

Oke, T.: Initial guidance to obtain representative meteorological observations at urban sites, Instruments and Observing Methods, Report no. 81, World Meteorological Organization, WMO/TDNo. 1250, 2006.

Olsson, J.: Evaluation of a scaling cascade model for temporal rain-fall disaggregation, Hydrol. Earth Syst. Sci., 2, 19-30, doi:10.5194/hess-2-19-1998, 1998.

Over, T. M. and Gupta, V. K.: A space-time theory of mesoscale rainfall using random cascades, J. Geophys. Res., 101, 26319 26331, 1996.

Paulson, K. S. and Baxter, P. D.: Downscaling of rain gauge time series by multiplicative beta cascade, J. Geophys. Res., 112, D09105, doi:10.1029/2006JD007333, 2007.

Peng, S., Piao, S., Ciais, P., Friedlingstein, P., Ottle, C., Bréon, F.M., Nan, H., Zhou, L., and Myneni, R. B.: Surface urban heat island across 419 global big cities, Environ. Sci. Technol., 46, 696-703, doi:10.1021/es2030438, 2012.

Robert, C. P.: Simulation of truncated normal variables, Stat. Comput., 5, 121-125, 1995.

Rupp, D. E., Keim, R. F., Ossiander, M., Brugnach, M., and Selker, J. S.: Time scale and intensity dependency in multiplicative cascades for temporal rainfall disaggregation, Water Resour. Res., 45, W07409, doi:10.1029/2008WR007321, 2009.

Rupp, D. E., Licznar, P., Adamowski, W., and Leśniewski, M.: Multiplicative cascade models for fine spatial downscaling of rainfall: parameterization with rain gauge data, Hydrol. Earth Syst. Sci., 16, 671-684, doi:10.5194/hess-16-671-2012, 2012.

Schmitt, T. G.: ATV-DVWK Kommentar, ATV-A 118 Hydraulische Berechnung von Entwässerungssystemen, DWA, Hennef, 2000.

Thames Tideway Tunnel (TTT): Needs Report, Appendix B, Report on Approaches to UWWTD Compliance in Relation to CSO's in 
major cities across the EU, available at: http://aim.prepared-fp7. eu/viewer/doc.aspx?id=28 (last access: 6 January 2015), 2010.

Willems, P.: Multidecadal oscillatory behaviour of rainfall extremes, Clim. Chang., 120, 931-944, doi:10.1007/s10584-0130837-x, 2013.
World Meteorological Organization (WMO): WMO-No. 8, Guide to Meteorological Instruments and Methods of Observation, 7 edition, World Meteorological Organization, Geneva, Switzerland , 2012. 